\title{
AN ÉTALE COHOMOLOGY DUALITY THEOREM FOR NUMBER FIELDS WITH A REAL EMBEDDING
}

\author{
MEL BIENENFELD
}

\begin{abstract}
The restriction on 2-primary components in the Artin-Verdier duality theorem [2] has been eliminated by Zink [9], who has shown that the sheaf of units for the étale topology over the ring of integers of any number field acts as a dualizing sheaf for a modified cohomology of sheaves. The present paper provides an alternate means of removing the 2-primary restriction. Like Zink's, it involves a topology which includes infinite primes, but it avoids modified cohomology and will be more directly applicable in the proof of a theorem of Lichtenbaum regarding zeta- and $L$-functions $[4,5]$. Related results-including the cohomology of units sheaves, the norm theorem, and punctual duality theorem of Mazur [6]-are also affected by the use of a topology including the infinite primes. The corresponding results in the new setting are included here.
\end{abstract}

Introduction. The Artin-Verdier duality theorem for number fields [2] has the complication that the 2-primary part of the groups involved must be ignored unless the number field is totally imaginary. In applications of the theorem it has therefore been necessary to make the appropriate qualifications. In particular, Lichtenbaum [4] uses the theorem to provide a cohomological description of the behavior at zero of Artin $L$-functions, but points out the possibility that his results are only true "up to a finite 2-torsion group" in the nontotally imaginary case.

Zink has proven a version of the duality theorem which takes care of the 2-primary difficulties [9], and with the proper adaptations Zink's theorem could be used to prove the Lichtenbaum result in full generality. However, several auxiliary results, not all explicitly stated in the Zink paper, are also used in that proof, which is soon to appear [5]. In addition, Lichtenbaum's theorem requires cohomology computations for the so-called "Artin-Verdier topology" which includes complex infinite primes where Zink only includes real ones. This makes no difference for the duality theorem, but is essential in studying the $L$-functions.

In the present paper we prove a version of the duality theorem which, though less general than Zink's, is stated in a form more directly applicable to proving Lichtenbaum's theorem. This version, here derived from Zink's, was originally proven independently, but unpublished [3]. (It has the additional virtue of explicitly including the 2-primary part of the isomorphism between the Galois group of the Hilbert class field and the Picard group.)

Received by the editors July $27,1986$.

1980 Mathematics Subject Classification (1985 Revision). Primary 11R34, 14F20; Secondary 11R37.

Key words and phrases. Artin-Verdier duality theorem, ètale cohomology, real algebraic number field. 
We also include the computations and auxiliary results regarding the Artin-Verdier topology which are used in [5]. Many of the techniques rely on Mazur's proof of the duality theorem [6] as well as Zink's, and on background material on Grothendieck topologies found in [1].

Throughout the paper, $K$ will denote an algebraic number field, $A$ its ring of integers, and $X=\operatorname{Spec} A$. The notation $\bar{K}, \bar{L}$, etc., will denote the algebraic closures of the fields $K, L$, etc., and $G_{K}, G_{L}$, etc., will denote the Galois groups of $\bar{K}$ over $K$, etc. $H^{p}(K, \cdot)$ denotes the group cohomology of $G_{K} \cdot G_{m}$ stands for the sheaf of units on any scheme.

If $U$ is an open subscheme of $X$, the category $\mathscr{S}_{U}$ is the sheaf category for the étale topology on $U$; i.e., the topology of finite étale morphisms in which a covering is a finite surjective family of morphisms. $\operatorname{Hom}_{U}(\cdot, \cdot), H^{p}(U, \cdot)$, etc., are functors on $\mathscr{S}_{U}$.

The inclusion map $j: \operatorname{Spec} K \rightarrow U$ induces the functors $j_{*}: G_{K}$-modules $\rightarrow \mathscr{S}_{U}$ and $j^{*}: \mathscr{S}_{U} \rightarrow G_{K}$-modules; $j^{*}$ is exact and left adjoint to $j_{*} ; j_{*}$ is left exact and preserves injectives [1, p. 42].

1. The Artin-Verdier topology and its sheaf cohomology. Let $X_{\infty}$ denote the set of infinite primes of $K$ (real embeddings and conjugate pairs of complex embeddings). We define the étale topology on $X_{\infty}$ to consist of maps of finite sets to $X_{\infty}$. A morphism in this topology is a set map over $X_{\infty}$, and a covering is a finite surjective family of morphisms. The sheaf category $\mathscr{S}_{X_{\infty}}$ is a direct sum of categories, one for each point in $X_{\infty}$; a sheaf $G$ for this topology is simply a collection of Abelian groups $\left\{G_{x}: x_{i} \in X_{\infty}\right\}$.

Let $x_{1}, \ldots, x_{t}$ be the elements of $X_{\infty}$. For each real $x_{i}$, fix an embedding of $\bar{K}$ into $\mathbb{C}$ which extends $x_{i}$, and let $\hat{K}_{i}$ denote the subfield of $\bar{K}$ mapped into $\mathbb{R}$ under this embedding. For each complex $x_{i}$, let $\hat{K}_{i}=\bar{K}$. Put $I_{i}=\operatorname{Gal}\left(\bar{K} / \hat{K}_{i}\right)$ for $i=$ $1, \ldots, t .\left(I_{i}=\{1\}\right.$ if $x_{i}$ is complex.)

If $U$ is an open subscheme of $X$, let $\tilde{U}$ be the set $U \sqcup X_{\infty}$. We are going to define the sheaf category $\mathscr{S}_{\tilde{U}}$. A sheaf $S$ on $\tilde{U}$ is a triple $S=\left(G, F,\left\{\sigma_{i}: 1 \leqslant i \leqslant t\right\}\right)$ where $F$ is a sheaf for the étale topology on $U, G$ is a sheaf on $X_{\infty}$, and each $\sigma_{i}$ : $G_{x_{i}} \rightarrow\left(j^{*} F\right)^{I_{i}}$ is a group homomorphism. ( $I_{i}$ acts on the $G_{K^{\prime}}$-module $j^{*} F$ as a subgroup.) A map of sheaves from $\left(G, F,\left\{\sigma_{i}\right\}\right)$ to $\left(G^{\prime}, F^{\prime},\left\{\sigma_{i}^{\prime}\right\}\right)$ is a pair of maps $\left(G \rightarrow G^{\prime}, F \rightarrow F^{\prime}\right)$ which commutes with the "specialization maps" $\left\{\sigma_{i}\right\},\left\{\sigma_{i}^{\prime}\right\}$.

This is the same definition of $\mathscr{S}_{\dot{U}}$ as in [9], except that we have included the complex infinite primes as well as the real ones. We reproduce from that paper two results that follow from the definition. The inclusion of the complex primes entails nothing new at this stage in the proofs.

Proposition 1.1. There are functors

$$
\mathscr{S}_{U} \stackrel{\stackrel{\varphi^{4}}{\rightarrow}}{\stackrel{\varphi^{4}}{\leftarrow}} \mathscr{S}_{\tilde{U}} \stackrel{\stackrel{\kappa^{*}}{\rightarrow}}{\stackrel{\kappa_{*}}{\leftarrow}} \mathscr{S}_{X_{\varkappa}}
$$


such that

(a) each functor is left adjoint to the one below it;

(b) $\phi_{!}, \phi^{*}, \kappa^{*}$ and $\kappa_{*}$ are exact;

(c) $\phi_{*}$ and $\kappa^{!}$are left exact; and

(d) $\phi^{*}, \phi_{*}, \kappa_{*}$ and $\kappa^{!}$preserve injectives. Explicitly,

$$
\begin{aligned}
\phi_{!} F & =\left(0, F,\left\{0_{i}\right\}\right), \\
\phi^{*} S & =F, \\
\phi_{*} F & =\left(\left\{\left(j^{*} F\right)^{I_{i}}\right\}, F,\left\{\operatorname{id}\left(j^{*} F\right)^{I_{i}}\right\}\right), \\
\kappa^{*} S & =G, \\
\kappa_{*} G & =\left(G, 0,\left\{0_{i}\right\}\right), \\
\kappa ! S & =\left\{\operatorname{ker} \sigma_{i}\right\} .
\end{aligned}
$$

For each inclusion $x_{i} \in X_{\infty}$ there are functors

$$
\mathscr{S}_{\tilde{U}} \stackrel{\stackrel{\kappa_{i}^{*}}{\stackrel{\kappa_{i *}}{\leftarrow}}}{\stackrel{\kappa_{i}^{\prime}}{\rightarrow}} \mathscr{S}_{x_{i}}=\mathscr{A} b
$$

such that properties (a) through (d) remain true with $\kappa$ replaced by $\kappa_{i}$.

$$
\begin{aligned}
& \kappa_{i}^{*} S=G_{x_{i}}, \\
& \kappa_{i *}\left(G_{x_{i}}\right)=\left(\left\{0, \ldots, G_{x_{i}}, \ldots, 0\right\}, 0,\left\{0_{i}\right\}\right), \\
& \kappa_{i}^{!} S=\operatorname{ker} \sigma_{i} .
\end{aligned}
$$

Finally, every object in $\mathscr{S}_{\tilde{U}}$ embeds in an injective, enabling us to define right derived functors. (Injectives in $\mathscr{S}_{\tilde{U}}$ are of the form $\phi_{*} J_{1} \oplus \kappa_{*} J_{2}$, where $J_{1}$ and $J_{2}$ are injectives in $\mathscr{S}_{U}$ and $\mathscr{S}_{X_{\infty}}$, respectively.)

The second result identifies $\mathscr{S}_{\tilde{U}}$ with the category of sheaves for the Grothendieck topology $E t_{\tilde{U}}$ (the Artin-Verdier topology). $E t_{\tilde{U}}$ is defined using the topology $T$ as follows:

Connected objects in $T$ are pairs $(V, M)$, where $V$ is an open subscheme of the spectrum of the ring of integers of a number field and $M$ is a subset of the infinite primes of the quotient field of $V$.

A morphism of connected objects $\pi:\left(V_{1}, M_{1}\right) \rightarrow\left(V_{2}, M_{2}\right)$ is a scheme map $V_{1} \rightarrow V_{2}$ which maps $M_{1}$ to $M_{2}$; such a morphism is étale if both $V_{1} \rightarrow V_{2}$ and $M_{1} \rightarrow M_{2}$ are unramified (in particular if a prime in $M_{1}$ is complex, so is its image in $M_{2}$ ).

Arbitrary objects and morphisms in $T$ come from connected ones by taking finite disjoint unions.

Coverings in $T$ are finite families of morphisms which are surjective on both finite and infinite primes. 
If we identify $\tilde{U}$ with the object $\left(U, X_{\infty}\right)$ of $T$ we can define $E t_{\tilde{U}}$ to be the étale morphisms over $\tilde{U}$ in the category $T$. The morphisms and coverings in $E t_{\tilde{U}}$ are then the obvious ones. We then have

Proposition 1.2 (SEE [9, 1.3.3]). (a) $T$ has fibered products, and the product of étale morphisms consists of étale morphisms (so that $T$ and $E t_{\tilde{U}}$ are indeed Grothendieck topologies);

(b) The category $\mathscr{S}_{\dot{U}}$ defined above is equivalent to the category of sheaves for $E t_{\dot{U}}$.

(c) Under this equivalence the functors $\phi_{*}$ and $\kappa_{*}\left(\right.$ resp. $\phi^{*}$ and $\left.\kappa^{*}\right)$ can be identified with the direct image (resp. pullback) functors induced by the inclusions $\phi: U \rightarrow \tilde{U}$ and $\kappa: X_{\infty} \rightarrow \tilde{U}$.

We now turn to cohomology. One way to define a cohomology functor on $\mathscr{S}_{\dot{U}}$ is to write $H^{p}(\tilde{U}, S)=\operatorname{Ext}_{U}^{\mathcal{D}}(\mathbb{Z}, S)$. Note that the constant sheaf $\mathbb{Z}$ on $\tilde{U}$ (i.e. constant on components when $\tilde{U}$ is considered as the final object of $\left.E t_{\tilde{U}}\right)$ is the same as the sheaf $\phi_{*} \mathbb{Z}$ induced from $U$. Examining the functor $\operatorname{Hom}_{\tilde{U}}\left(\phi_{*} \mathbb{Z}, \cdot\right)$ in the "triple" category, it is easy to see that the $H^{p}$ are the same as the derived functors of

$$
S \rightarrow F(U) \times \times_{j^{*} F} G_{x_{1}} \times_{j^{*} F} G_{x_{2}} \times_{j^{*} F} \cdots \times \times_{j^{*} F} G_{x_{t}}
$$

for the sheaf $S=\left(\left\{G_{x_{i}}\right\}, F,\left\{\sigma_{i}\right\}\right)$, which is Zink's definition. $H^{0}(\tilde{U}, S)$ is precisely the group of global sections $S(\tilde{U})$.

For any sheaf $F$ on $U$ there is an exact sequence in $\mathscr{S}_{\dot{U}}$

$$
0 \rightarrow \phi_{!} F \rightarrow \phi_{*} F \rightarrow \kappa_{*} \kappa^{*} \phi_{*} F \rightarrow 0 .
$$

(Use Proposition 1.1 or $[1,2.6$, p. 69].) We apply this sequence to $F=\mathbb{Z}$ to derive the local cohomology sequence for $X_{\infty} \subset \tilde{U}$. From Proposition 1.1 we have $\kappa_{*} \kappa^{*} \phi_{*} \mathbb{Z}$ $=\oplus_{i=1}^{i} \kappa_{i *} \mathbb{Z}$. We define

$$
H_{x_{i}}^{p}(\tilde{U}, S)=R^{p} \kappa_{i}^{\prime} S
$$

Applying the long exact sequence of $\operatorname{Ext}_{\tilde{U}}(\cdot, S)$ to $(1.3)$ we obtain

Proposition 1.4 (SEe [1, III, 2.9 AND 9, 2.2.1]). There is an exact sequence

$$
\cdots \rightarrow \bigoplus_{i=1}^{t} H_{x_{i}}^{p}(\tilde{U}, S) \rightarrow H^{p}(\tilde{U}, S) \rightarrow H^{p}\left(U, \phi^{*} S\right) \rightarrow \bigoplus_{i=1}^{t} H_{x_{i}}^{p+1}(\tilde{U}, S) \rightarrow \cdots
$$

Proof. We need to show $\operatorname{Ext}_{\tilde{U}}^{p}\left(\kappa_{*} \kappa * \mathbb{Z}, S\right) \cong \oplus H_{x_{i}}^{p}(\tilde{U}, S)$ and $\operatorname{Ext}_{\tilde{U}}^{p}(\phi, \mathbb{Z}, S) \cong$ $H^{p}\left(U, \phi^{*} S\right)$. But $\left.\operatorname{Ext}_{\tilde{U}}^{p}=\oplus \kappa_{i *} \mathbb{Z}, S\right)=\oplus \operatorname{Ext}_{\tilde{U}}^{p}\left(\kappa_{i *} \mathbb{Z}, S\right)$ and $\operatorname{Ext}_{\tilde{U}}^{p}\left(\kappa_{i *} \mathbb{Z}, S\right)$ is the $p$ th derived functor of $\operatorname{Hom}_{\tilde{U}}\left(\kappa_{i *} \mathbb{Z}, S\right)$ which is isomorphic to $\operatorname{Hom}\left(\mathbb{Z}, \kappa_{i}^{\vdots} S\right)=\kappa_{i}^{\vdots} S$ by Proposition 1.1. Also $\operatorname{Ext}_{\tilde{U}}^{p}(\phi, \mathbb{Z}, S) \cong \operatorname{Ext}_{\tilde{U}}^{p}\left(\mathbb{Z}, \phi^{*} S\right)$ by the adjunction and exactness of $\phi$, and $\phi^{*}$.

Two useful lemmas regarding cohomology on $S_{\tilde{U}}$ follow.

Lemma 1.5. A sheaf $S$ on $\tilde{U}$ such that $\phi^{*} S=0$ is said to be concentrated (or punctual) at infinite primes. If $S$ is such a sheaf, $H^{p}(\tilde{U}, S)=0$ for $p>0$.

Proof. $S=\kappa_{*} G$, where $G=\kappa^{*} S$. By exactness and adjunction of $\kappa^{*}$ and $\kappa_{*}$, $H^{p}(\tilde{U}, S)=\operatorname{Ext}_{\tilde{U}}^{p}\left(\phi_{*} \mathbb{Z}, \kappa_{*} G\right) \cong \operatorname{Ext}_{X_{x}}^{p}\left(\kappa^{*} \phi_{*} \mathbb{Z}, G\right) \cong \oplus \operatorname{Ext}^{p}\left(\mathbb{Z}, G_{x_{1}}\right)$. This is zero unless $p=0$, and $H^{0}(\tilde{U}, S)=\oplus_{i=1}^{t} G_{x_{i}}$. 
LeMMA 1.6. Let $\alpha: \operatorname{Spec} K \rightarrow \tilde{U}$ denote the composite $\phi \circ j$. Then $\alpha$ induces $a$ morphism of topologies. If $M$ is a $G_{K}$-module there is a Leray spectral sequence

$$
H^{p}\left(\tilde{X}, R^{q} \alpha_{*} M\right) \Rightarrow H^{p+q}(K, M) \text {. }
$$

Proof. Such a sequence exists if $\alpha_{*}$ is left exact and preserves injectives. Both $\phi_{*}$ and $j_{*}$ have these properties. Noting that $\alpha_{*}, \phi_{*}$ and $j_{*}$ are all direct image functors, it is clear that $\alpha_{*}=\phi_{*} \circ j_{*}$, and we are done.

We close the section with the basic results on base extension.

Definition 1.8. Let $L$ be a finite extension of $K, B$ the ring of integers of $L$, and $V$ an open subscheme of $Y=\operatorname{Spec} B$. Let $j$ denote either inclusion $j$ : Spec $K \rightarrow U$ or $j: \operatorname{Spec} L \rightarrow V$. Similarly put $\phi: U \rightarrow \tilde{U}$ and $\phi: V \rightarrow \tilde{V}$, and $\kappa: X_{\infty} \rightarrow \tilde{U}$ and $\kappa$ : $Y_{\infty} \rightarrow \tilde{V}$. Denote by $\pi$ both natural projections $\pi: V \rightarrow U$ and $\pi: Y_{\infty} \rightarrow X_{\infty}$, and write $\pi: \tilde{V} \rightarrow \tilde{U}$ both as a summary of these data and as andication that we may consider our sheaf categories as categories for the étale topologies on $\tilde{V}$ and $\tilde{U}$. The projections induce morphisms of topologies (by fibered products), hence pairs of adjoint functors

$$
\mathscr{S}_{U} \stackrel{\pi^{*}}{\rightarrow} \mathscr{S}_{V} \quad \mathscr{S}_{X_{\infty}} \stackrel{\pi_{*}^{*}}{\stackrel{\pi_{*}^{*}}{\leftarrow}} \mathscr{S}_{Y_{\infty}}
$$

We wish to combine these into an adjoint pair

$$
\mathscr{S}_{\tilde{U}}^{\stackrel{\pi^{*}}{\rightarrow}} \mathscr{S}_{\tilde{V}}
$$

For each $y \in Y_{\infty}$, choose $I_{y}$ so that if $\pi(y)=x$ then $I_{Y} \subseteq I_{x}$ (i.e. $I_{y}=I_{x}$ if $I_{y}$ is nontrivial). Write $\tau_{\tilde{U}}: \mathscr{S}_{U} \rightarrow \mathscr{S}_{x_{\infty}}$ for the functor $\tau_{\tilde{U}} F=\left\{\left(j^{*} F\right)^{I_{x}}: x \in X_{\infty}\right\}$, and $\tau_{\tilde{V}}: \mathscr{S}_{V} \rightarrow \mathscr{S}_{Y_{\infty}}$ for $\tau_{\tilde{V}} F=\left\{\left(j^{*} F\right): y \in Y_{\infty}\right\}$.

LEMMA 1.9. There is a canonical isomorphism of functors $\theta_{*}: \pi_{*} \tau_{\bar{V}} \rightarrow \tau_{\bar{U}} \pi_{*}$.

Proof. For $F \in \mathscr{S}_{V}$, we evaluate $\pi_{*} \tau_{\tilde{V}} F$ and $\tau_{\tilde{U}} \pi_{*} F$ at a prime $x \in X_{\infty}$ :

$$
\begin{gathered}
\pi_{*} \tau_{\tilde{V}} F(x)=\bigoplus_{y / x} \tau_{\tilde{V}} F(y)=\bigoplus_{y / x}\left(j^{*} F\right)^{I_{v}}, \\
\tau_{\tilde{U}} \pi_{*} F(x)=\left(j^{*} \pi_{*} F\right)^{I_{x}} .
\end{gathered}
$$

If $j^{*} F$ is the $G_{L}$-module $N$, then $j^{*} \pi_{*} F$ is its direct image sheaf on Spec $K$. As a $G_{K}$-module this is the induced module $M_{G_{K}}^{G_{L} N}$ [7, p. I-12]. If $\Sigma=\left\{g_{1}, \ldots, g_{n}\right\}$ is a set of left coset representations for $G_{L}$ in $G_{K}$, for any $g \in G_{K}$ and any $g_{i} \in \Sigma$ we have $g g_{i}=g_{j} h$ for a unique $j$ and $h \in G_{L}$. We may write $M_{G_{K}}^{G_{L}} N$ as (the Abelian group) $\oplus_{\Sigma} N$, where $g \in G_{K}$ acts by sending the element whose $i$ th component is $n_{i}$ to the element whose $i$ th component is $h n_{j}$, with $h$ and $j$ as above.

As for $\bigoplus_{y / x}\left(j^{*} F\right)^{I_{y}}=\bigoplus_{y / x} N^{I_{y}}$, choose $y_{0} / x$ and for each pair $g_{i}, g_{j} \in \Sigma$ such that $g_{i} y_{0}=g_{j} y_{0}$ (i.e., such that $x$ is real, $g_{i} y_{0}$ is complex, and $g_{j} y_{0}$ as a field embedding agrees with $g_{i} y_{0}$ up to conjugation), choose only one of $\left\{g_{i}, g_{j}\right\}$, yielding a subset $T \subseteq \Sigma$. Then

$$
\bigoplus_{y / x}\left(j^{*} F\right)^{I_{y}} \cong \bigoplus_{g_{i} \in T} N^{I_{g_{1}\left(y_{0}\right)}} .
$$


One then checks that the map $\left\{n_{i}: g_{i} \in \Sigma\right\} \rightarrow\left\{n_{i}: g_{i} \in T\right\}$ gives us our isomorphism $\pi_{*} \tau_{\tilde{V}} F \rightarrow \tau_{\tilde{U}} \pi_{*} F$.

The corresponding situation for $\pi^{*}$ is somewhat different. We will define a natural transformation $\theta^{*}: \pi^{*} \tau_{\tilde{U}} \rightarrow \tau_{\tilde{V}} \pi^{*}$ which is paired with $\theta_{*}$. Choose $F \in \mathscr{S}_{U}$ and let $F \rightarrow \pi_{*} \pi^{*} F$ be the map corresponding to the identity on $\pi^{*} F$ under the adjunction map. Apply $\tau_{\tilde{U}}$ and compose with $\theta_{*}^{-1}$ to get

$$
\tau_{\tilde{U}} F \rightarrow \tau_{\tilde{U}} \pi_{*} \pi^{*} F \rightarrow \pi_{*} \tau_{\tilde{V}} \pi^{*} F .
$$

Using the adjunction property for $\mathscr{S}_{X_{x}}$ and $\mathscr{S}_{Y_{x}}$ on this composite yields our map $\theta^{*}$. In general, $\theta^{*}$ is not an isomorphism.

We are now able to define $\pi^{*}$ and $\pi_{*}$ on the mapping cylinder (triples) categories quite formally. If $\mathscr{A}_{1}$ and $\mathscr{A}_{2}$ are any Abelian categories, and $\tau_{\mathscr{A}}: \mathscr{A}_{2} \rightarrow \mathscr{A}_{1}$ any left exact functor, put $\mathscr{A}=$ the category of triples $A=\left(A_{1} \in \mathscr{A}_{1}, A_{2} \in \mathscr{A}_{2}, \alpha\right.$ : $A_{1} \rightarrow \tau_{\mathscr{A}} A_{2}$ ). Similarly if $\mathscr{B}_{1}, \mathscr{B}_{2}$ and $\tau_{\mathscr{B}}: \mathscr{B}_{2} \rightarrow \mathscr{B}_{1}$ are as above, let $\mathscr{B}$ be the category consisting of objects $B=\left(B_{1}, B_{2}, \beta: B_{1} \rightarrow \tau_{\mathscr{B}} B_{2}\right)$. If $p_{1}: A_{1} \rightarrow B_{1}$ and $p_{2}: A_{2} \rightarrow B_{2}$ are any functors, and there is a natural transformation $\theta: p_{1} \tau_{\mathscr{A}} \rightarrow \tau_{\mathscr{B}} p_{2}$, then $p_{\theta}: \mathscr{A} \rightarrow \mathscr{B}$ is defined by

$$
p\left(A_{1}, A_{2}, \alpha\right)=\left(p_{1} A_{1}, p_{2} A_{2}, p_{1} A_{1} \stackrel{p_{1} \alpha}{\rightarrow} p_{1} \tau_{\mathscr{A}} \mathscr{B} 1 \stackrel{\theta}{\rightarrow} \tau_{\mathscr{B}} p_{2} B_{2}\right) .
$$

Proposition 1.10. In the above situation, if $p_{1}$ and $p_{2}$ are both left exact (resp. right exact, resp. exact), then so is $p_{\theta}$.

The proof is a formal exercise involving the definitions of kernel, cokernel, etc. Note that the result of the proposition is independent of the specific transformation $\theta$.

PROPOSITION 1.11. In the same setting assume there are adjoint pairs

$$
\mathscr{A}_{1} \stackrel{p_{\llcorner *}^{*}}{\rightarrow} \mathscr{B}_{1} \quad \text { and } \quad \mathscr{A}_{2} \stackrel{p^{*}}{\rightarrow} \mathscr{p}_{p_{2 *}}^{\rightarrow} \text {. }
$$

Assume $\theta_{*}: p_{1 *} \tau_{\mathscr{B}} \rightarrow \tau_{\mathscr{A}} p_{2 *}$ is an isomorphism of functors, and $\theta^{*}$ is defined using $\theta_{*}$ as above. Then $p^{*}\left(=p_{\theta^{*}}^{*}\right)$ and $p_{*}\left(=p_{* \theta_{*}}\right)$ are an adjoint pair.

Proof. For $A \in \mathscr{A}$ and $B \in \mathscr{B}$ we require a pair of naturally defined inverses $\operatorname{Hom}_{\mathscr{B}}\left(p^{*} A, B\right) \rightleftarrows \operatorname{Hom}_{\mathscr{A}}\left(A, p_{*} B\right)$. An element of the group on the left consists of maps $p_{1}^{*} A_{1} \rightarrow B_{1}$ and $p_{2}^{*} A_{2} \rightarrow B_{2}$ such that the diagram

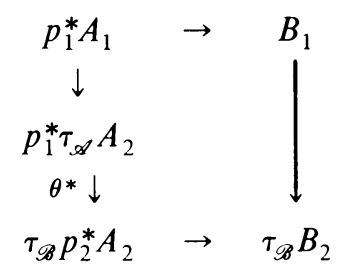

commutes. The adjunction properties in our data give maps $A_{1} \rightarrow p_{1 *} B_{1}$ and $A_{2} \rightarrow p_{2 *} B_{2}$; in order for this to produce an element of $\operatorname{Hom}_{\mathscr{A}}\left(A, p_{*} B\right)$ we must 
check that

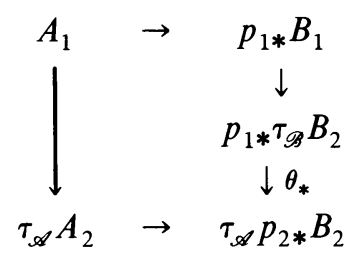

is commutative. But the composite

$$
A_{1} \rightarrow p_{1 *} B_{1} \rightarrow p_{1 *} \tau_{\mathscr{B}} B_{2}
$$

is paired under the adjunction of $p_{1}^{*}$ and $p_{1 *}$ with

$$
p_{1}^{*} A_{1} \rightarrow B_{1} \rightarrow \tau_{\mathscr{B}} B_{2}
$$

of the given data. This is the same as

$$
p_{1}^{*} A_{1} \rightarrow \tau_{\mathscr{B}} p_{2}^{*} A_{2} \rightarrow \tau_{\mathscr{B}} B_{2}
$$

by the definition of maps in $\mathscr{B}$. Expanding the definition of $p_{1}^{*} A_{1} \rightarrow \tau p_{2}^{*} A_{2}$, we replace $(1.16)$ by

$$
p_{1}^{*} A_{1} \rightarrow p_{1}^{*} \tau_{\mathscr{A}} A_{2} \stackrel{\theta^{*}}{\rightarrow} \tau_{\mathscr{A}} p_{2}^{*} A_{2} \rightarrow \tau_{\mathscr{B}} B_{2}
$$

Now reverse the pairing to discover that (1.14) is the same as

$$
A_{1} \rightarrow \tau_{\mathscr{A}} A_{2} \rightarrow p_{1 *} \tau_{\mathscr{A}} p_{2}^{*} A_{2} \rightarrow p_{1 *} \tau_{\mathscr{B}} B_{2} .
$$

By definition of $\theta^{*}$, the middle map here is

$$
\tau_{\mathscr{A}} A_{2} \rightarrow \tau_{\mathscr{A}} p_{2 *} p_{2}^{*} A_{2} \stackrel{\theta_{*}^{-1}}{\rightarrow} p_{1 *} \tau_{\mathscr{B}} p_{2}^{*} A_{2}
$$

But the diagram

$$
\begin{array}{ccc}
\tau_{\mathscr{A}} p_{2 *} p_{2}^{*} A_{2} & \rightarrow & \tau_{\mathscr{A}} p_{2 *} B_{2} \\
\downarrow \theta_{*}^{-1} & & \downarrow \theta_{*}^{-1} \\
p_{1 *} \tau_{\mathscr{B}} p_{2}^{*} A_{2} & \rightarrow & p_{1 *} \tau_{\mathscr{B}} B_{2}
\end{array}
$$

commutes, so we may replace $(1.18)$ by

$$
A_{1} \rightarrow \tau_{\mathscr{A}} A_{2} \rightarrow \tau_{\mathscr{A}} p_{2 *} p_{2}^{*} A_{2} \rightarrow \tau_{\mathscr{A}} p_{2}^{*} B_{2} \stackrel{\theta_{*}^{-1}}{\rightarrow} p_{1 *} \tau_{\mathscr{B}} B_{2}
$$

Applying the adjunction of $p_{2}^{*}$ and $p_{2 *}$ to the two middle maps reduces this to

$$
A_{1} \rightarrow \tau_{\mathscr{A}} A_{2} \rightarrow \tau_{\mathscr{A}} p_{2}^{*} B_{2} \stackrel{\theta_{*}^{-1}}{\rightarrow} p_{1 *} \tau_{\mathscr{B}} B_{2}
$$

where the middle map now is that given by the data. Following this by $\theta_{*}$ completes one direction in diagram (1.13) and shows that it is indeed equal to the other direction.

Defining the inverse map $\operatorname{Hom}_{\mathscr{A}}\left(A, p_{*} B\right) \rightarrow \operatorname{Hom}_{\mathscr{B}}\left(p^{*} A, B\right)$ involves the commutativity of a similar diagram, and the proof there is similar. That the maps are actually inverses is trivial. 
We may now state our results for the categories $\mathscr{S}_{\tilde{U}}$ and $\mathscr{S}_{\tilde{V}}$.

Proposition 1.23. There is a pair of adjoint functors

$$
\mathscr{S}_{\tilde{U}}^{\stackrel{\pi^{*}}{\rightarrow}} \mathscr{S}_{\tilde{V}}
$$

such that $\phi^{*} \pi^{*}=\pi^{*} \phi^{*}, \phi^{*} \pi_{*}=\pi_{*} \phi^{*}, \kappa^{*} \pi^{*}=\pi^{*} \kappa^{*}$, and $\kappa^{*} \pi_{*}=\pi_{*} \kappa^{*}$, using the notation of (1.8). Both functors in (1.24) are exact.

Proof. After what we have done we need only show that $\pi^{*}$ and $\pi_{*}$ are exact on the component sheaf categories. Each $\pi^{*}$ is exact, and each $\pi_{*}$ left exact, by [1, p. 42 and Theorem 4.14, p. 45]. It remains to show that $\pi_{*}$ preserves epimorphisms in $\mathscr{S}_{V}$ and $\mathscr{S}_{Y_{x}}$. For $\mathscr{S}_{V}$ this is true because $\pi: V \rightarrow U$ is a finite morphism of schemes over which étales are separated [1, Corollary 4.1, p. 94]. For $\mathscr{S}_{Y_{x}, \pi_{*}}$ is simply the identity on each subcategory of sheaves concentrated at a single $y \in Y_{\infty}$, so exactness is clear.

2. Sheaves of units and constant sheaves. We now compute the cohomology of $\phi, G_{m}$ and $\phi_{*} G_{m}$ on $\tilde{U}$. There is an exact sequence in $\mathscr{S}_{\tilde{U}}$,

$$
0 \rightarrow G_{m} \rightarrow j_{*} G_{m} \rightarrow \coprod_{x \text { closed }} i_{*}^{x} \mathbb{Z} \rightarrow 0
$$

where $i^{x}$ denotes the inclusion $x \in U[1$, p. 103]. We extend this to $\tilde{U}$ in two ways,

$$
0 \rightarrow \phi ! G_{m} \rightarrow \alpha_{*} G_{m} \rightarrow \coprod_{x \in U} i_{*}^{x} \mathbb{Z} \oplus \coprod_{i=1}^{t} \kappa_{i *} \hat{K}_{i}^{*} \rightarrow 0
$$

and

$$
0 \rightarrow \phi_{*} G_{m} \rightarrow \alpha_{*} G_{m} \rightarrow \coprod_{x \in U} i_{*}^{x} \mathbb{Z} \rightarrow 0
$$

In (2.2) and (2.3) we are also using $i^{x}$ to denote the inclusion $x \rightarrow \tilde{U}$; note that $i_{*}^{x}=\phi_{*} i_{*}^{x}$ in the obvious sense. Sequence (2.2) is exact on $X_{\infty}$ because the second and third sheaves agree there, while $\kappa^{*} \phi ! G_{m}=0 ;(2.3)$ is exact on $X_{\infty}$ because $\kappa^{*} \phi_{*} G_{m}=\kappa^{*} \alpha_{*} G_{m}$ and the third sheaf has no $X_{\infty}$-component.

Zink computes the cohomology of $\alpha_{*} G_{m}$ in dimensions up to 3; we would like to go further and require the following theorem.

THEOREM 2.4. [8]. Let $L$ be a number field. For each infinite prime $v$ of $L$ let $L_{v}$ denote the completion of $L$ at $v$. Then the natural map $H^{p}\left(L, G_{m}\right) \rightarrow \amalg_{\text {all }} H^{p}\left(L_{v}, G_{m}\right)$ is a surjection for $p=2$ and an isomorphism for $p>2$. More explicitly, for $p>2$, $H^{p}\left(L, G_{m}\right)$ is 0 if $p$ is odd and $(\mathbb{Z} / 2 \mathbb{Z})^{r}$ if $p$ is even (here $r$ is the number of real primes of $L)$.

PROPOSITION 2.5 (SEE [9, 2.5.7]).

$$
H^{p}\left(\tilde{U}, \alpha_{*} G_{m}\right)= \begin{cases}K^{*}, & p=0 \\ 0, & p=1 \\ \operatorname{Br}_{0} K, & p=2 \\ 0, & p>2\end{cases}
$$


The group $\mathrm{Br}_{0} K$ denotes the Brauer classes of $K$ with zero invariants at all (real) infinite primes.

Proof. We use spectral sequence (1.7). As in $[9,2.5]$, we discover $R^{p} \alpha_{*} G_{m} \cong$ $\oplus_{x_{i} \text { real }} \kappa_{i_{*}} H^{p}\left(I_{i}, \bar{K}^{*}\right)$ for $p \geqslant 1$. Since this is concentrated at infinite primes, its cohomology is zero in positive dimensions and (1.7) degenerates to the exact sequence.

$$
\begin{aligned}
0 & \rightarrow H^{1}\left(\tilde{U}, \alpha_{*} G_{m}\right) \rightarrow H^{1}\left(K, G_{m}\right) \rightarrow \oplus H^{1}\left(I_{i}, \bar{K}^{*}\right) \\
& \rightarrow H^{2}\left(\tilde{U}, \alpha_{*} G_{m}\right) \rightarrow H^{2}\left(K, G_{m}\right) \rightarrow \cdots .
\end{aligned}
$$

Combining this with Hilbert's Theorem 90 , the relationship of the Brauer group to local invariants, and Corollary 2.2, yields Proposition 2.5.

Proposition 2.6.

$$
H^{p}\left(\tilde{U}, \amalg i_{*}^{x} \mathbb{Z}\right)= \begin{cases}\bigoplus_{x \in U} \mathbb{Z}, & p=0, \\ 0, & p=1, \\ \bigoplus_{x \in U} \mathbb{Q} / \mathbb{Z}=\oplus \operatorname{Br} K_{x}, & p=2, \\ 0, & p>2 .\end{cases}
$$

$K_{x}$ denotes completion at $x$.

The computation in $[6,2.3]$ suffices here for all dimensions.

We put this information, together with the fact that $H^{p}\left(\tilde{U}, \amalg \kappa_{i *} \hat{K}_{i}^{*}\right)$ is 0 for $p>0$, into the long exact cohomology sequences of (2.2) and (2.3) to obtain

Proposition 2.7. (a) If $S=X \backslash U$ is nonempty,

$$
H^{p}\left(\tilde{U}, \phi_{*} G_{m}\right)= \begin{cases}A_{S}^{*}, & p=0, \\ \operatorname{Pic} A_{S}, & p=1, \\ \left\{\text { Elements of } \mathrm{Br}_{0} K \text { with no invariants in } U\right\}, & p=2, \\ 0, & p>2\end{cases}
$$

(b)

$$
H^{p}\left(\tilde{X}, \phi_{*} G_{m}\right)= \begin{cases}A^{*}, & p=0 \\ \operatorname{Pic} A, & p=1 \\ 0, & p=2 \\ \mathbb{Q} / \mathbb{Z}, & p=3 \\ 0, & p>3\end{cases}
$$

(c) If $\nu: K^{*} \rightarrow \amalg \mathbb{Z} \oplus \amalg K_{i}^{*}$ is defined by valuation at finite primes and the identity at infinite primes,

$$
H^{p}\left(\tilde{X}, \phi ! G_{m}\right)= \begin{cases}0, & p=0, \\ \left(\amalg \mathbb{Z} \oplus \amalg K_{i}^{*}\right) / \nu\left(K^{*}\right), & p=1, \\ 0, & p=2, \\ \mathbb{Q} / \mathbb{Z}, & p=3, \\ 0, & p>3 .\end{cases}
$$


Thus $H^{1}\left(\tilde{X}, \phi, G_{m}\right)$ is isomorphic to a dense subgroup of the ideles of $K$ modulo unit idèles and principal idèles.

We also compute the cohomology of $G_{m}$ on $U$ so that we have this statement for the nontotally imaginary case. The technique is very similar to that used in proving the last two propositions.

To find $H^{p}\left(U, j_{*} G_{m}\right)$ we use the spectral sequence

$$
H^{p}\left(U, R^{q} j_{*} G_{m}\right) \Rightarrow H^{p+q}\left(K, G_{m}\right)
$$

coming from the fact that $j_{*}$ is left exact and preserves injectives. Recall that $R^{q} j_{*} G_{m}$ is the associated sheaf to the presheaf $P^{q}$ defined by $P^{q}(V)=H^{q}\left(L, G_{m}\right)$ where $L$ is the function field of $V$ (see [1, Corollary 4.7, p. 42]). For $q>0$ all stalks of the presheaf vanish, because every positive-dimensional Galois cocycle over a local field splits in some unramified extension (see $[6,2.1])$. This implies that $R^{q} j_{*} G_{m}=0$ [1, III, 1.8]).

The sequence (2.8) thus degenerates to the isomorphisms $H^{p}\left(U, j_{*} G_{m}\right) \cong$ $H^{p}\left(K, G_{m}\right)$. The cohomology of $\amalg i_{*}^{x} \mathbb{Z}$ on $U$ is the same as it is on $\tilde{U}$, so we are ready to find $H^{p}\left(U, G_{m}\right)$ from the long exact sequence of $(2.1)$.

Proposition 2.9. Let $r_{1}$ be the number of real primes of $K$. Then

(a) If $S=X \backslash U$ is nonempty,

$$
H^{p}\left(U, G_{m}\right)= \begin{cases}A_{S}^{*}, & p=0, \\ \operatorname{Pic} A_{S}, & p=1, \\ \{\text { Elements of BrK with no invariants in } U\}, & p=2, \\ 0, & p>2, p \text { odd }, \\ (\mathbb{Z} / 2 \mathbb{Z})^{r_{1}}, & p>2, p \text { even } .\end{cases}
$$

(b)

$$
H^{p}\left(X, G_{m}\right)= \begin{cases}A^{*}, & p=0, \\ \operatorname{Pic} A, & p=1, \\ \left\{\text { Elements of }(\mathbb{Z} / 2 \mathbb{Z})^{r_{1}} \text { with } 0 \text { component sum }\right\}, & p=2, \\ \mathbb{Q} / \mathbb{Z}, & p=3, \\ (\mathbb{Z} / 2 \mathbb{Z})^{r_{1}}, & p>3, p \text { even }, \\ 0, & p>3, p \text { odd } .\end{cases}
$$

Proof. The long exact sequence of (2.1) is

$$
\begin{aligned}
0 \rightarrow H^{0}\left(U, G_{m}\right) \rightarrow K^{*} \rightarrow \coprod_{u \text { closed }} \mathbb{Z} \rightarrow H^{1}\left(U, G_{m}\right) \rightarrow 0 \\
0 \rightarrow H^{2}\left(U, G_{m}\right) \rightarrow \operatorname{Br} K \rightarrow \coprod_{u \text { closed }} \mathbb{Q} / \mathbb{Z} \rightarrow H^{3}\left(U, G_{m}\right) \rightarrow H^{3}\left(K, G_{m}\right) \rightarrow 0, \\
H^{q}\left(U, G_{m}\right) \cong H^{1}\left(K, G_{m}\right), \quad q>3 .
\end{aligned}
$$


REMARK 2.11. Note that the isomorphism $H^{3}\left(\tilde{X}, \phi_{*} G_{m}\right) \cong \mathbb{Q} / \mathbb{Z}$ of Proposition 2.7(b) can be defined by lifting an element arbitrarily to $H^{2}\left(\tilde{X}, \amalg i_{*}^{x} \mathbb{Z}\right)$, which is canonically isomorphic to $\oplus \mathbb{Q} / \mathbb{Z}$, and following this by the sum map

$$
\begin{array}{ccccc}
H^{2}\left(\tilde{X}, \amalg i_{*}^{x} \mathbb{Z}\right) & \rightarrow & H^{3}\left(\tilde{X}, \phi_{*} G_{m}\right) & \rightarrow & 0 \\
\downarrow \cong & & \downarrow \cong & & \\
\oplus \mathbb{Q} / \mathbb{Z} & \stackrel{\text { sum }}{\rightarrow} & \mathbb{Q} / \mathbb{Z} & \rightarrow & 0
\end{array}
$$

The corresponding procedure for $H^{3}\left(X, G_{m}\right)$, however, does not work unless $K$ is totally imaginary. For the corresponding diagram to commute, the sum map would have to be replaced by $2 \cdot$ sum.

We now use 2.7(b) to compute the values of the Ext functors which will appear in our duality theorem, for the special case of the constant sheaf $\mathbb{Z} / n \mathbb{Z}$. The long exact sequence of $\operatorname{Ext}_{\tilde{X}}\left(\cdot, \phi_{*} G_{m}\right)$ applied to the sequence $0 \rightarrow \mathbb{Z} \stackrel{n}{\rightarrow} \mathbb{Z} \rightarrow \mathbb{Z} / n \mathbb{Z} \rightarrow 0$ gives part (a) of the following result:

Proposition 2.13. (a)

$$
\operatorname{Ext}_{\tilde{X}}\left(\mathbb{Z} / n \mathbb{Z}, \phi_{*} G_{m}\right)= \begin{cases}\mu_{n}(A), & p=0, \\ \operatorname{Pic} A / n \operatorname{Pic} A, & p=2, \\ \mathbb{Z} / n \mathbb{Z}, & p=3, \\ 0, & p>3 .\end{cases}
$$

There is an exact sequence $0 \rightarrow A^{*} / n A^{*} \rightarrow \operatorname{Ext}_{\tilde{X}}^{1}\left(\mathbb{Z} / n \mathbb{Z}, \phi_{*} G_{m}\right) \rightarrow n \operatorname{Pic} A \rightarrow 0$. (Compare [6, 2.4, Remark (C)]. The corresponding functors on $X$ give different results when $n$ is even and $K$ is not totally imaginary.)

(b)

$$
H^{1}(\tilde{X}, \mathbb{Z} / n \mathbb{Z}) \cong \operatorname{Hom}(\operatorname{Pic} A, \mathbb{Z} / n \mathbb{Z}) .
$$

Proof of (b). We use 1.6 applied to $\mathbb{Z} / n \mathbb{Z}$ as a $G_{K}$-module. We find an exact sequence

$$
0 \rightarrow H^{1}(\tilde{X}, \mathbb{Z} / n \mathbb{Z}) \rightarrow H^{1}(K, \mathbb{Z} / n \mathbb{Z}) \rightarrow H^{0}\left(\tilde{X}, R^{1} \phi_{*} \mathbb{Z} / n \mathbb{Z}\right)
$$

and require the value of $R^{1} \phi_{*} \mathbb{Z} / n \mathbb{Z}$. If $V \rightarrow \tilde{X}$ is an étale (possibly including infinite primes) with function field $L$, let $L_{V}$ be the maximal extension of $L$ unramified at primes of $V$. Then the presheaf $P(V)=H^{1}\left(G_{L}, \mathbb{Z} / n \mathbb{Z}\right)=\operatorname{Hom}\left(G_{L}, \mathbb{Z} / n \mathbb{Z}\right)$ has the associated sheaf $P^{\sharp}(V)=\operatorname{Hom}\left(G_{L_{v}}, \mathbb{Z} / n \mathbb{Z}\right)$. (It is easy to check that this definition satisfies the sheaf axiom, and that the natural map $P \rightarrow P^{\#}$ induces an isomorphism on stalks.) This shows that $R^{1} \phi_{*} \mathbb{Z} / n \mathbb{Z}(\tilde{X})=\operatorname{Hom}\left(G_{K^{\prime}}, \mathbb{Z} / n \mathbb{Z}\right)$, where $K^{\prime}$ is the Hilbert class field of $K$, and (2.14) becomes

$$
0 \rightarrow H^{1}(\tilde{X}, \mathbb{Z} / n \mathbb{Z}) \rightarrow \operatorname{Hom}\left(G_{K}, \mathbb{Z} / n \mathbb{Z}\right) \rightarrow \operatorname{Hom}\left(G_{K^{\prime}}, \mathbb{Z} / n \mathbb{Z}\right) .
$$

But $G_{K} / G_{K^{\prime}}=\operatorname{Gal}\left(K^{\prime} / K\right) \cong \operatorname{Pic} A$, and the result follows.

3. The norm map. If $Y=\operatorname{Spec} B$, where $B / A$ is an integral extension of number rings, $\pi: Y \rightarrow X$ is the natural projection, $L$ is the quotient field of $B$, and $G_{m, Y}$ and $G_{m, X}$ are the respective multiplicative groups, Mazur defines the norm map from $\pi_{*} G_{m, Y}$ to $G_{m, X}$ as a map of sheaves on $X[6,2.7]$. If $j: \operatorname{Spec} K \rightarrow X$ or $j$ : Spec $L \rightarrow Y$ denotes either of the natural inclusions, an analogous definition 
provides a norm map from $\pi_{*} j_{*} G_{m}$ to $j_{*} G_{m}$. Using the properties of cokernels, there is a unique map, which we shall also call a norm, from $\pi_{*}\left(\amalg_{y \text { closed in } Y} i_{*} \mathbb{Z}\right)$ to $\amalg_{x \text { closed in } x} i_{*}^{x} \mathbb{Z}$ such that the diagram

$$
\begin{array}{llll}
0 \rightarrow & \pi_{*} G_{m} \rightarrow & \pi_{*} j G_{m} \rightarrow & \pi_{*}\left(\amalg i^{y}{ }^{*} \mathbb{Z}\right) \rightarrow 0 \\
& \downarrow \text { norm } & \downarrow \text { norm } & \downarrow \text { norm } \\
0 \rightarrow & G_{m} \rightarrow & j_{*} G_{m} \rightarrow & \amalg_{x} i_{*}^{x} \mathbb{Z} \rightarrow 0
\end{array}
$$

commutes. We also have, for any sheaf $F$ on $Y$, the natural map $\nu: H^{p}\left(X, \pi_{*} F\right) \rightarrow$ $H^{p}(Y, F)$ which is isomorphism for all $p$ because $\pi_{*}$ is exact. The composite (norm) $\cdot \nu^{-1}$ thus defines a map $N$ of long exact cohomology sequences

$$
\begin{array}{cccc}
\cdots H^{p}\left(Y, G_{m}\right) & \rightarrow H^{p}\left(Y, j_{*} G_{m}\right) & \rightarrow H^{p}\left(Y, \amalg i_{*}^{*} \mathbb{Z}\right) & \rightarrow H^{p+1}\left(Y, G_{m}\right) \cdots \\
\downarrow N & \downarrow N & \downarrow N & \\
\cdots H^{p}\left(X, G_{m}\right) & \rightarrow H^{p}\left(X, j_{*} G_{m}\right) & \rightarrow H^{p}\left(X, \amalg i_{*}^{x} \mathbb{Z}\right) & \rightarrow H^{p+1}\left(X, G_{m}\right) \cdots
\end{array}
$$

If we identify $H^{p}\left(X, j_{*} G_{m}\right)$ with $H^{p}\left(K, G_{m}\right)$ and $H^{p}\left(Y, j_{*} G_{m}\right)$ with $H^{p}\left(L, G_{m}\right)$, then $N$ is just the corestriction map of group cohomology [7, p. I-13] because $\pi_{*} j_{*} G_{m}$ corresponds to the induced $G_{K}$-module $M_{G_{K}}^{G_{L}} \bar{K}^{*}$ [7, p. I-12] and the maps $\nu$ and (norm) correspond to the maps coming from Shapiro's lemma and the map $M_{G_{K}}^{G_{l}} \bar{K}^{*} \rightarrow \bar{K}^{*}$ defing the corestriction.

We may examine the map (norm): $\pi_{*} \amalg_{y} i_{*} \mathbb{Z} \rightarrow \amalg_{x} i_{*}^{x} \mathbb{Z}$ locally; that is, as a collection of maps, for each closed point $y$ in $Y$ lying over $x$ in $X$ : (norm): $\pi_{*} i_{*}^{y} \mathbb{Z} \rightarrow i_{*}^{x} \mathbb{Z}$. Let $k$ be the residue field of $X$ at $x$, and let $l$ be the residue field of $Y$ at $y$. The decomposition lemma [1, Corollary 2.5], which identifies $\mathscr{S}_{X}$ with a "triples" or "mapping cylinder" category, allows us to identify this as a map of $G_{k}$-modules. As a $G_{k}$-module, $\pi_{*} i_{*} \mathbb{Z}$ corresponds to

$$
\begin{aligned}
\underset{U \text { covering } x}{\lim _{*}} i_{*}^{y} \mathbb{Z}\left(U \times_{X} Y\right) & =\underset{C / A \underset{\text { anramified }}{\rightarrow}}{\lim } i_{*}^{y} \mathbb{Z}\left(\operatorname{Spec}\left(C \otimes_{A} B\right)\right) \\
& =\mathbb{Z}\left(\operatorname{Spec}\left(\bar{k} \otimes_{k} l\right)\right)=\bigoplus_{G(l / k)} \mathbb{Z}
\end{aligned}
$$

and the $G_{k}$-action on both factors of the tensor product induces the action on $\oplus_{G(l / k)} \mathbb{Z}$ of the induced module $M_{G_{k}}^{G_{l}} \mathbb{Z}$. Pulling the norm map back to $\pi_{*} j_{*} G_{m}$ and restricting to étales covering $x$, we see that the map induced after valuation at $y$ is the natural map of $G_{k}$-modules $M_{G_{k}}^{G} \mathbb{Z} \rightarrow \mathbb{Z}$. On positive-dimension cohomology groups, the map $N: H^{p}\left(Y, i_{*}^{y} \mathbb{Z}\right) \rightarrow H^{p}\left(X, i_{*}^{x} \mathbb{Z}\right)$ can therefore also be identified with the corestriction

$$
\text { cor: } H^{p}\left(G_{l}, \mathbb{Z}\right) \rightarrow H^{p}\left(G_{k}, \mathbb{Z}\right)
$$

The diagram

$$
\begin{array}{ccc}
H^{p}\left(Y, j_{*} G_{m}\right) & \rightarrow & H^{p}\left(Y, i_{*}^{y} \mathbb{Z}\right) \\
\downarrow N & & \downarrow N \\
H^{p}\left(X, j_{*} G_{m}\right) & \rightarrow & H^{p}\left(Y, i_{*}^{x} \mathbb{Z}\right)
\end{array}
$$


is thus replaced by

$$
\begin{array}{ccc}
H^{p}\left(L, G_{m}\right) & \rightarrow & H^{p}\left(G_{l}, \mathbb{Z}\right) \\
\downarrow \text { cor } & & \downarrow \text { cor } \\
H^{p}\left(K, G_{m}\right) & \rightarrow & H^{p}\left(G_{k}, \mathbb{Z}\right)
\end{array}
$$

where the horizontal arrows come from restriction to the respective decomposition groups followed by valuation. For $p=2$ this square becomes

$$
\begin{array}{ccc}
\operatorname{Br}(L) & \stackrel{\text { inv }_{y}}{\rightarrow} & \mathbb{Q} / \mathbb{Z} \\
\downarrow N & & \downarrow 1 \\
\operatorname{Br}(K) & \stackrel{\text { inv }_{x}}{\rightarrow} & \mathbb{Q} / \mathbb{Z} .
\end{array}
$$

To relate this to $H^{3}\left(Y, G_{m}\right)$ and $H^{3}\left(X, G_{m}\right)$ we have

$$
\begin{aligned}
& \operatorname{Br}(L) \stackrel{\amalg^{\text {inv }_{y}}}{\rightarrow} \quad \coprod_{y} \mathbb{Q} / \mathbb{Z} \quad \rightarrow \quad H^{3}\left(Y, G_{m}\right) \rightarrow 0 \\
& \downarrow N \quad \downarrow \amalg_{y / x} 1 \quad \downarrow N \\
& \operatorname{Br}(K) \stackrel{\amalg^{\text {inv }_{x}}}{\rightarrow} \quad \coprod_{x} \mathbb{Q} / \mathbb{Z} \quad \rightarrow \quad H^{3}\left(X, G_{m}\right) \rightarrow 0 .
\end{aligned}
$$

If $K$ and $L$ are both totally imaginary, Remark 2.11 shows that the norm map $N$ : $H^{3}\left(Y, G_{m}\right) \rightarrow H^{3}\left(X, G_{m}\right)$ induces the identity under the identifications with $\mathbb{Q} / \mathbb{Z}$. However, if $L$ is totally imaginary and $K$ is not, the diagram

$$
\begin{array}{ccc}
H^{3}\left(Y, G_{m}\right) & \cong \mathbb{Q} / \mathbb{Z} \\
\downarrow N & \downarrow 1 \\
H^{3}\left(X, G_{m}\right) & \cong & \mathbb{Q} / \mathbb{Z}
\end{array}
$$

does not commute, because, for example, the norm annihilates the image of any element of $\oplus_{y \in Y \text { closed }} \mathbb{Q} / \mathbb{Z}$ the sum of whose components is $\frac{1}{2}$. In this case $N$ induces multiplication by 2 on $\mathbb{Q} / \mathbb{Z}$.

There is no problem in extending the norm map to $\mathscr{S}_{\tilde{X}}$ and $\mathscr{S}_{\tilde{Y}}$. Apply $\phi_{*}$ to (3.1) to get maps $\phi_{*} \pi_{*} G_{m} \rightarrow \phi_{*} G_{m}, \phi_{*} \pi_{*} j_{*} G_{m} \rightarrow \alpha_{*} G_{m}$, and $\phi_{*} \pi_{*}\left(\mathrm{U} i_{*}^{y} \mathbb{Z}\right) \rightarrow \phi_{*}\left(\mathrm{U} i_{*}^{x} \mathbb{Z}\right)$. Note that $\pi_{*}: \mathscr{S}_{\tilde{V}} \rightarrow \mathscr{S}_{\tilde{U}}$ from 1.23 satisfies $\pi_{*} \phi_{*}=\phi_{*} \pi_{*}$ (pull both composites back to $\mathscr{S}_{Y}$ and $\mathscr{S}_{Y_{\infty}}$ ). By adjunction and exactness of $\pi^{*}$ and $\pi_{*}$ we may define $\nu$ : $H^{p}\left(\tilde{X}, \pi_{*} F\right) \stackrel{\cong}{\rightarrow} H^{p}(\tilde{Y}, F)$ for any $F \in \mathscr{S}_{\tilde{Y}}$, thus giving us the norm map on cohomology for $\phi_{*} G_{m}, \alpha_{*} G_{m}$, and $\amalg \phi_{*} i_{*}^{x} \mathbb{Z}$. Repeating the previous computations using the cohomology sequence of (2.3), we find

Proposition 3.2. Under the natural identifications, the diagram

$$
\begin{array}{ccc}
H^{3}\left(\tilde{Y}, \phi_{*} G_{m}\right) & \cong & \mathbb{Q} / \mathbb{Z} \\
\downarrow N & & \downarrow 1 \\
H^{3}\left(\tilde{X}, \phi_{*} G_{m}\right) & \cong \mathbb{Q} / \mathbb{Z}
\end{array}
$$

commutes, regardless of whether $K$ and $L$ are totally imaginary. 
With slight abuse of notation we will also denote by $N$ the composite map

$$
\operatorname{Ext}_{\tilde{Y}}^{p}\left(F, \phi_{*} G_{m}\right) \rightarrow \operatorname{Ext}_{\tilde{X}}^{p}\left(\pi_{*} F, \pi_{*} \phi_{*} G_{m}\right) \stackrel{\text { norm }}{\rightarrow} \operatorname{Ext}_{\tilde{X}}^{p}\left(\pi_{*} F, \phi_{*} G_{m}\right),
$$

where the first map is induced by the exact functor $\pi_{*}$. The fact that (3.3) commutes enables us to establish

Proposition 3.5. The diagram

$$
\begin{array}{cccccc}
H^{p}(\tilde{Y}, F) & \times & \operatorname{Ext}_{\tilde{Y}}^{3-p}\left(F, \phi_{*} G_{m}\right) & \rightarrow & H^{3}\left(\tilde{Y}, \phi_{*} G_{m}\right) \\
\uparrow \nu & & & \downarrow N & & \downarrow N \\
H^{p}\left(\tilde{X}, \pi_{*} F\right) & \times & \operatorname{Ext}_{\tilde{X}}^{3-r}\left(\pi_{*} F, \phi_{*} G_{m}\right) & \rightarrow & H^{3}\left(\tilde{X}, \phi_{*} G_{m}\right)
\end{array}
$$

is a commutative diagram of pairings into $\mathbb{Q} / \mathbb{Z}$. More precisely, for $\alpha \in H^{p}\left(\tilde{X}, \pi_{*} F\right)$ and $\beta \in \operatorname{Ext}_{\tilde{X}}^{3-p}\left(F, \phi_{*} G_{m}\right)$, if $\langle\cdot, \cdot\rangle$ denotes both pairings then

(a) $N(\langle\nu(\alpha), \beta\rangle)=\langle\alpha, N(\beta)\rangle$,

(b) $N: H^{3}\left(\tilde{Y}, \phi_{*} G_{m}\right) \rightarrow H^{3}\left(\tilde{X}, \phi_{*} G_{m}\right)$ is the identity on $\mathbb{Q} / \mathbb{Z}$.

Proof (SeE [6, 2.7]). Part (a) simply follows from the definitions, functorial properties and the naturality of the pairing; part (b) comes from diagram (3.3).

The "norm theorem," however, $[6,2.7]$ breaks down for general extensions $\pi$ : $\tilde{Y} \rightarrow \tilde{X}$. That is, $N$ is not always an isomorphism. The problem arises in the case of punctual sheaves concentrated at complex primes of $\tilde{Y}$ lying over real primes of $\tilde{X}$. We investigate what can be said about such sheaves, and reproduce the following lemma of Zink [9, 2.3]:

LEMma 3.7. If $S=\left(\left\{G_{x_{i}}\right\}, F,\left\{\sigma_{i}\right\}\right)$ is a sheaf on $\tilde{U}$, and $\kappa_{i}: x_{i} \rightarrow \tilde{X}$ is the inclusion of an infinite prime, then

$$
\begin{aligned}
& R^{0} \kappa_{i} S=\operatorname{ker} \sigma_{i}, \\
& R^{1} \kappa_{i} S=\operatorname{coker} \sigma_{i}, \\
& R^{q} \kappa_{i} S=H^{q-1}\left(I_{i}, j^{*} F\right), \quad q \geqslant 2,
\end{aligned}
$$

where $I_{i}$ is as defined in $\$ 1$.

The extension of Zink's result to the case of complex $x_{i}$ is trivial. Note that in this case $R^{q} \kappa_{i} S=0$ for $q \geqslant 2$.

LEMMA 3.8. Let $M$ be an Abelian group. If $x_{i}$ is complex, $\operatorname{Ext}_{\tilde{X}}^{p}\left(\kappa_{i *} M, \phi_{*} G_{m}\right)=0$ for all $p$. If $x_{i}$ is real there are isomorphisms

$$
\operatorname{Ext}_{\tilde{X}}^{p}\left(\kappa_{i *} M, \phi_{*} G_{m}\right)= \begin{cases}0, & p<3, \\ \operatorname{Hom}(M, \mathbb{Z} / 2 \mathbb{Z}), & p \geqslant 3, p \text { odd }, \\ \operatorname{Ext}^{1}(M, \mathbb{Z} / 2 \mathbb{Z}), & p>3, p \text { even } .\end{cases}
$$

Proof. There is a composite functor spectral sequence

$$
\operatorname{Ext}^{p}\left(M, R^{q_{\kappa}} \phi_{i} \phi_{*} G_{m}\right) \Rightarrow \operatorname{Ext}_{\tilde{X}}^{p+q}\left(\kappa_{i *} M, \phi_{*} G_{m}\right)
$$

coming from the adjunction of $\kappa_{i *}$ and $\kappa_{i}^{!}$. By 3.7, $R^{q} \kappa_{i}^{!} \phi_{*} G_{m}$ is zero for $q<2$. For $q \geqslant 2, R^{q} \kappa_{i}^{\prime} \phi_{*} G_{m}=H^{q-1}\left(G_{\hat{K}_{i}}, \bar{K}^{*}\right)$. This is zero unless $x_{i}$ is real and $q$ is odd, in which case $H^{q-1}\left(G_{\hat{K}}, \bar{K}^{*}\right)=\mathbb{Z} / 2 \mathbb{Z}$. The terms $E_{2}^{p, q}$ of the spectral sequence are 0 
for $p>1$ because these Ext functors are on the category of Abelian groups. For $q \geqslant 2$, only one of $E_{2}^{0, q}$ or $E_{2}^{1, q-1}$ is nonzero, depending on whether $q$ is odd or even. The composite Ext is therefore isomorphic to one of these.

Lemma 3.8 prepares us for our results regarding the norm:

Proposition 3.10. Let $\pi: \tilde{Y} \rightarrow \tilde{X}$ be as in 3.1, and let $y$ be an infinite prime of $\tilde{Y}$ which is unramified over $\tilde{X}$ (i.e., $y$ and $\pi(y)$ are either both real or both complex). Let $P=\kappa_{y *} M$ be $a$ sheaf on $\tilde{Y}$ concentrated at $y$. Then $N: \operatorname{Ext}_{\tilde{Y}}^{p}\left(P, \phi_{*} G_{m}\right) \rightarrow$ $\operatorname{Ext}_{\tilde{X}}^{p}\left(\pi_{*} P, \phi_{*} G_{m}\right)$ is an isomorphism for all $p$.

Proof. If $y$ is complex, both groups are zero, so assume $y$ is real. We may also assume $p>2$. We replace the map $\kappa_{y}$ with $\kappa: Y_{\infty} \rightarrow \tilde{Y}$ and rewrite sequence (3.9) considering $M$ as a sheaf on $Y_{\infty}$ :

$$
\operatorname{Ext}_{Y_{\infty}}^{p}\left(M, R^{q} \kappa^{\prime} \phi_{*} G_{m}\right) \Rightarrow \operatorname{Ext}_{\tilde{Y}}^{p+q}\left(\kappa_{*} M, \phi_{*} G_{m}\right) .
$$

The value of $R^{q} \kappa^{\prime} \phi_{*} G_{m}$ at a point in $Y_{\infty}$ depends on whether that point is real or complex.

Consider the corresponding sequence after applying $\pi_{*}$ :

$$
\operatorname{Ext}_{X_{x}}^{p}\left(\pi_{*} M, R^{q} \kappa^{\prime} \pi_{*} \phi_{*} G_{m}\right) \Rightarrow \operatorname{Ext}_{\tilde{X}}^{p+q}\left(\pi_{*} \kappa_{*} M, \pi_{*} \phi_{*} G_{m}\right) .
$$

The sheaf $\pi_{*} M$ on $X_{\infty}$ is concentrated at the prime $x$ below $y$. Lemma 3.3 identifies the value of $R^{q}{ }^{\prime} \pi_{*} \phi_{*} G_{m}$ with $H^{q-1}\left(\hat{K}_{x}, M_{G_{K}}^{G_{L}} \bar{K}^{*}\right)$. This is $\oplus_{\mathrm{Gal}(L / K)} \mathbb{Z} / 2 \mathbb{Z}$ for even $q$, using the fact that $x$ splits completely in $L$. On the other hand, $\pi_{*}$ commutes with $R^{q} \kappa^{!}$on $\tilde{X}$ and on $\tilde{Y}$. (This is true for $q=0$ using the isomorphism $\theta_{*}$ of (1.9); for higher $q$ use that $\pi_{*}$ is exact and preserves injectives.) Changing the position of $\pi_{*}$ in (3.12) gives a commutative diagram

$$
\begin{array}{ccc}
\operatorname{Hom}_{Y_{\infty}}\left(M, R^{q} \kappa^{\prime} \phi_{*} G_{m}\right) & \cong & \operatorname{Ext}_{\tilde{Y}}^{q}\left(\kappa_{*} M, \phi_{*} G_{m}\right) \\
\downarrow \pi_{*} & & \downarrow \pi_{*} \\
\operatorname{Hom}_{X_{\infty}}\left(\pi_{*} M, \pi_{*} R^{q} \kappa^{\prime} \phi_{*} G_{m}\right) & \cong & \operatorname{Ext}_{\tilde{X}}^{q}\left(\pi_{*} \kappa_{*} M, \pi_{*} \phi_{*} G_{m}\right)
\end{array}
$$

for even $q$, and the corresponding diagram for odd $q$. The vertical map on the left is the map $\operatorname{Hom}(M, \mathbb{Z} / 2 \mathbb{Z}) \rightarrow \operatorname{Hom}\left(M, \oplus_{\pi^{-1}(x)} \mathbb{Z} / 2 \mathbb{Z}\right)$ induced by the injection of $\mathbb{Z} / 2 \mathbb{Z}$ into the component of the direct sum corresponding to $y$.

To determine the effect of the norm on the sheaves in the second variable on the bottom row of (3.13) recall that over the generic point the norm is the map $M_{G_{K}}^{G_{l}} \bar{K}^{*} \rightarrow \bar{K}^{*}$ corresponding to corestriction. Taking even-dimensional cohomology as $G_{\hat{K}_{x}}$-modules gives the sum map $\bigoplus_{\pi^{-1}(x)} \mathbb{Z} / 2 \mathbb{Z} \rightarrow \mathbb{Z} / 2 \mathbb{Z}$. For even $q$ there is a commutative diagram

$$
\begin{array}{rlc}
\operatorname{Hom}_{X_{\infty}}\left(\pi_{*} M, \pi_{*} R^{q} \kappa^{\prime} \phi_{*} G_{m}\right) & \cong & \operatorname{Ext}_{\tilde{X}}^{q}\left(\pi_{*} \kappa_{*} M, \pi_{*} \phi_{*} G_{m}\right) \\
\downarrow \text { sum } & & \downarrow \text { norm } \\
\operatorname{Hom}_{X_{x}}\left(\pi_{*} M, R^{q} \kappa^{\prime} \phi_{*} G_{m}\right) & \cong & \operatorname{Ext}_{\tilde{X}}^{q}\left(\pi_{*} \kappa_{*} M, \phi_{*} G_{m}\right) .
\end{array}
$$


The composite of (3.13) and (3.14) is simply the map

$$
\operatorname{Hom}(M, \mathbb{Z} / 2 \mathbb{Z}) \stackrel{y \text {-component }}{\rightarrow} \operatorname{Hom}(M, \oplus \mathbb{Z} / 2 \mathbb{Z}) \stackrel{\text { sum }}{\rightarrow} \operatorname{Hom}(M, \mathbb{Z} / 2 \mathbb{Z}),
$$

which is the identity; this completes the proof for even $q$.

For odd $q$, simply consider the functors in (3.13) and (3.14) to be functors on $M$. Since the composite is an isomorphism for any $M$ with $q$ even, and since $\kappa_{*}$ preserves injectives, we may shift dimensions.

The following version of the norm theorem holds:

THEOREM 3.15. Let $F$ be a constructible sheaf on $Y$. Then $N: \operatorname{Ext}_{\tilde{Y}}^{p}\left(\phi_{*} F, \phi_{*} G_{m}\right) \rightarrow$ $\operatorname{Ext}_{\tilde{X}}^{p}\left(\pi_{*} \phi_{*} F, \phi_{*} G_{m}\right)$ is an isomorphism for $0 \leqslant p \leqslant 2$. If, in addition, all primes of $Y_{\infty}$ are unramified over $\tilde{X}, N$ is an isomorphism for all $p$.

Proof. We use the decompositions

$$
\begin{gathered}
0 \rightarrow \phi_{!} F \rightarrow \phi_{*} F \rightarrow \kappa_{*} \kappa^{*} \phi_{*} F \rightarrow 0, \\
0 \rightarrow \phi_{!} \pi_{*} F \rightarrow \pi_{*} \phi_{*} F \rightarrow \kappa_{*} \kappa^{*} \pi_{*} \phi_{*} F \rightarrow 0,
\end{gathered}
$$

recalling that $\pi_{*}$ commutes with the other functors. For $p<3$, $\operatorname{Ext}_{\tilde{Y}}^{p}\left(\kappa_{*} \kappa^{*} \phi_{*} F, \phi_{*} G_{m}\right)=\operatorname{Ext}_{\tilde{X}}^{p}\left(\pi_{*} \kappa_{*} \kappa^{*} \phi_{*} F, \phi_{*} G_{m}\right)=0$, and for $p \geqslant 3, N$ is an isomorphism on $\operatorname{Ext}_{\tilde{Y}}^{p}\left(\kappa_{*} \kappa^{*} F, \phi_{*} G_{m}\right)$ under our additional hypothesis by 3.10. We are thus reduced to the norm on $\operatorname{Ext}_{\tilde{Y}}^{p}\left(\phi, F, \phi_{*} G_{m}\right)=\operatorname{Ext}_{Y}^{p}\left(F, G_{m}\right)$, which is an isomorphism according to the norm theorem of Mazur.

We also investigate the map $N$ on $\operatorname{Ext}_{\tilde{Y}}^{p}\left(\phi_{*} F, \alpha_{*} G_{m}\right)$ (recall $\left.\alpha_{*}=\phi_{*} j_{*}\right)$. Using the extension of the norm on $\pi_{*} j_{*} G_{m}$, we get norm: $\pi_{*} \alpha_{*} G_{m} \rightarrow \alpha_{*} G_{m}$ which provides

$$
N: \operatorname{Ext}_{\tilde{Y}}^{p}\left(\phi_{*} F, \alpha_{*} G_{m}\right) \rightarrow \operatorname{Ext}_{\tilde{X}}^{p}\left(\pi_{*} \phi_{*} F, \alpha_{*} G_{m}\right) .
$$

THEOREM 3.16. With $\phi_{*} G_{m}$ replaced by $\alpha_{*} G_{m}$, Theorem 3.15 remains true.

Proof. If $P$ is a sheaf concentrated at an infinite prime of $\tilde{Y}$, we claim there are isomorphisms $\operatorname{Ext}_{\tilde{Y}}^{p}\left(P, \phi_{*} G_{m}\right) \rightarrow \operatorname{Ext}_{\tilde{Y}}^{p}\left(P, \alpha_{*} G_{m}\right)$ and $\operatorname{Ext}_{\tilde{X}}^{p}\left(\pi_{*} P, \phi_{*} G_{m}\right) \rightarrow$ $\operatorname{Ext}_{\tilde{X}}^{p}\left(\pi_{*} P, \alpha_{*} G_{m}\right)$ which commute with the map $N$. For this we use sequence 2.3, and reduce to showing that $\operatorname{Ext}_{\tilde{Y}}^{p}\left(P, i_{*}^{w} \mathbb{Z}\right)=\operatorname{Ext}_{\tilde{X}}^{p}\left(\pi_{*} P, i_{*}^{v} \mathbb{Z}\right)=0$, where $i^{w}$ and $i^{v}$ are the inclusions of a prime of $Y$ into $\tilde{Y}$ and a prime of $X$ into $\tilde{X}$, respectively. However $i^{w *} P=i^{v *} \pi_{*} P=0$, so by the adjunction and exactness of $\left(i^{w *}, i_{*}^{w}\right)$ and $\left(i^{v *}, i_{*}^{v}\right)$, this proves the claim.

As in 3.15 , we are now reduced to $N: \operatorname{Ext}_{Y}^{p}\left(F, j_{*} G_{m}\right) \rightarrow \operatorname{Ext}_{X}^{p}\left(\pi_{*} F, j_{*} G_{m}\right)$. We prove this is an isomorphism as in the proof of the norm theorem in [6], by using the sequence $0 \rightarrow \rho_{1} p^{*} F \rightarrow F \rightarrow Q \rightarrow 0$ of sheaves on $Y$, where $\rho: V \rightarrow Y$ is an appropriate subscheme of $Y$ and $Q=\amalg_{y / V} i_{*}^{y} M_{y}$ is punctual. We show $\operatorname{Ext}_{Y}^{p}\left(i_{*}^{y} M_{y}, j_{*} G_{m}\right)$ is 0 . The right adjoint of $i_{*}^{y}$ is $i^{y !}$. Recall from Artin [1, p. 69] that $i^{y !}$ associates to a sheaf on $Y$ the subsheaf of sections with support on $y$. Since $i_{*}^{y}$ is exact we have the Leray spectral sequence

$$
\operatorname{Ext}_{y}^{p}\left[M_{y}, R^{q} i \cdot{ }^{v !}\left(j_{*} G_{m}\right)\right] \Rightarrow \operatorname{Ext}_{Y}^{p+q}\left(i_{*}^{y} M_{y}, j_{*} G_{m}\right)
$$

and we claim $R^{q} i^{v !}\left(j_{*} G_{m}\right)=0$ for all $q$. 
Note that the composite $i^{y !} j_{*}$ is the zero functor. Thus in the spectral sequence $R^{q}{ }^{y}{ }^{y}\left(R^{p} j_{*} G_{m}\right) \Rightarrow R^{q+p}\left(i^{y !} j_{*}\right) G_{m}$ the terms converge to 0 . We know from the proof of 2.6 that $R^{p} j_{*} G_{m}=0$ for $p>0$, so $R^{q} i{ }^{y}\left(j_{*} G_{m}\right)=0$ as well.

Since the same applies to $\operatorname{Ext}_{X}^{p}\left(\pi_{*} i_{*}^{y} M_{y}, j_{*} G_{m}\right)=\operatorname{Ext}_{X}^{p}\left(i_{*}^{x} \pi_{*} M_{y}, j_{*} G_{m}\right)$, where $\pi$ : $y \rightarrow x$ also represents the local projection onto a prime of $X$, we are finally reduced to the case (as in [6]) of an étale extension of subschemes whose function fields form a Galois extension. The map $F \rightarrow \pi^{*} \pi_{*} F$ that exists in this case provides an inverse to $N$ just as well for $j_{*} G_{m}$ as it does for $\alpha_{*} G_{m}$.

4. Punctual duality on $X$. Our version of the punctual duality theorem $[6,2.7(\mathrm{~d})]$ is not fully necessary in the proof of the general theorem which we give here. We prove punctual duality first because we wish to make explicit some of the relationships between categories that are involved.

Definition. Let $i^{x}: x \rightarrow \tilde{X}$ denote the inclusion of a closed point. A sheaf $P$ on $\tilde{X}$ is called punctual if there exists a finite set $W$ of closed points of $\tilde{X}$ such that $P=\amalg_{x \in W} i_{*}^{x} M_{x}$, where each $M_{x}$ is a sheaf on $x$.

Proposition 4.1. Let $x$ be a finite closed point of $\tilde{X}$ with residue field $k(x)$, and let $G^{x}=G_{k(x)}$. Let $M$ be a discrete $G^{x}$-module. Let $i: x \rightarrow \tilde{X}$ be the inclusion. Then for all $p$ there are isomorphisms

$$
\begin{gathered}
H^{p}\left(G^{x}, M\right) \cong H^{p}\left(\tilde{X}, i_{*} M\right), \\
\operatorname{Ext}_{x}^{p-1}(M, \mathbb{Z}) \cong \operatorname{Ext}_{\tilde{X}}^{p}\left(i_{*} M, \phi_{*} G_{m}\right),
\end{gathered}
$$

and

$$
H^{p-1}\left(G^{x}, \mathbb{Z}\right) \cong H^{p}\left(\tilde{X}, \phi_{*} G_{m}\right)
$$

such that the diagram

$$
\begin{array}{ccccc}
H^{p}\left(\tilde{X}, i_{*} M\right) & \times & \operatorname{Ext}_{\tilde{X}}^{3-p}\left(i_{*} M, \phi_{*} G_{m}\right) & \rightarrow & H^{3}\left(\tilde{X}, \phi_{*} G_{m}\right) \\
\mathfrak{1} \cong & & \hat{1} \cong & & 1 \cong \\
H^{p}\left(G^{x}, M\right) & \times & \operatorname{Ext}_{x}^{2-p}(M, \mathbb{Z}) & \rightarrow & H^{2}\left(G^{x}, \mathbb{Z}\right)
\end{array}
$$

commutes in the obvious sense (the lower line is in the category of discrete $G^{x}$-modules; negative-dimensional functors are zero by definition).

Proof (Lichtenbaum). Our first step is to replace $H^{p}\left(\tilde{X}, i_{*} M\right)=\operatorname{Ext}_{\tilde{X}}^{p}\left(\mathbb{Z}, i_{*} M\right)$ by $\operatorname{Ext}_{\tilde{X}}^{p}\left(i_{*} \mathbb{Z}, i_{*} M\right)$, and $H^{3}\left(\tilde{X}, \phi_{*} G_{m}\right)$ by $\operatorname{Ext}_{\tilde{X}}^{3}\left(i_{*} \mathbb{Z}, \phi_{*} G_{m}\right)$. We do this by shifting both Ext functors along the sequence $0 \rightarrow \rho_{1} \mathbb{Z} \rightarrow \mathbb{Z} \rightarrow i_{*} \mathbb{Z} \rightarrow 0$ of sheaves on $\tilde{X}$, where $\rho: \tilde{U} \rightarrow \tilde{X}$ is the inclusion of $\tilde{U}=\tilde{X} \backslash x$. The functor $\rho_{\text {! }}$ is defined using a category of triples which combines $\mathscr{S}_{\tilde{U}}$ with the category of $G^{x}$-modules and is isomorphic to $\mathscr{S}_{\tilde{X}}$ by the decomposition lemma. By exactness and adjunction of $\rho_{\text {! }}$ and $\rho^{*}$ we have $\operatorname{Ext}_{\tilde{X}}^{p}(\rho, \mathbb{Z}, F)=\operatorname{Ext}_{\tilde{U}}^{p}\left(\mathbb{Z}, \rho^{*} F\right)$ for any sheaf $F$. With $F=i_{*} M$, $\rho^{*} F=0$, so $\operatorname{Ext}_{\tilde{X}}^{p}(\mathbb{Z}, F)=\operatorname{Ext}_{\tilde{X}}^{p}\left(i_{*} \mathbb{Z}, F\right)$ for all $p$. With $F=\phi_{*} G_{m}$, we apply Proposition 2.7(a) to find that in the case of just one "missing" prime $H^{p}\left(\tilde{U}, \rho^{*} \phi_{*} G_{m}\right)=H^{p}\left(\tilde{U}, \phi_{*} G_{m}\right)=0$ for $p \geqslant 2$, and in particular $\operatorname{Ext}_{\tilde{X}}^{3}\left(\mathbb{Z}, \phi_{*} G_{m}\right)=$ $\operatorname{Ext}_{\tilde{X}}^{3}\left(i_{*} \mathbb{Z}, \phi_{*} G_{m}\right)$. 
The isomorphisms commute with the Yoneda pairings by the naturality of the pairings, so this step is complete.

We now replace $\operatorname{Ext}_{\bar{X}}^{3-p}\left(i_{*} M, \phi_{*} G_{m}\right)$ by $\operatorname{Ext}_{\bar{X}}^{2-p}\left(i_{*} M, i_{*} \mathbb{Z}\right)$ and $\operatorname{Ext}_{\tilde{X}}^{3}\left(i_{*} \mathbb{Z}, \phi_{*} G_{m}\right)$ by $\operatorname{Ext}_{\tilde{X}}^{2}\left(i_{*} \mathbb{Z}, i_{*} \mathbb{Z}\right)$. To do this we shift backwards in the second variable along the appropriate long exact Ext-sequences of

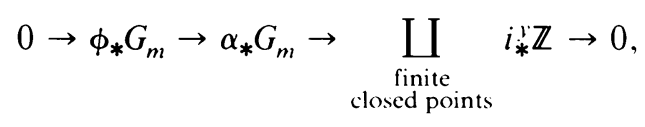

using the fact that Yoneda pairings commute with the coboundary maps on Ext sequences. We must show that $\operatorname{Ext}_{\tilde{X}}^{p}\left(i_{*} N, \alpha_{*} G_{m}\right)=0$ and $\operatorname{Ext}_{\tilde{X}}^{p}\left(i_{*} N, i_{*} \mathbb{Z}\right)=0$ for all discrete $G^{x}$-modules $N$ ( $N=\mathbb{Z}, M$ for our purposes), for all $p$, and for $y \neq x$. But if we denote by $i^{\prime}$ the inclusion $x \rightarrow X$, we can write $i_{*} N=\phi^{*} i_{*}^{\prime} N$, which is the same as $\phi, i_{*}^{\prime} N$ (use sequence (1.3)). By exactness and adjunction of $\phi$ ! and $\phi^{*}$ we are reduced to investigating

$$
\operatorname{Ext}_{X}^{p}\left(i_{*}^{\prime} N, \phi^{*} \alpha_{*} G_{m}\right)=\operatorname{Ext}_{X}^{p}\left(i_{*}^{\prime} N, j_{*} G_{m}\right)
$$

and $\operatorname{Ext}_{X}^{p}\left(i_{*}^{\prime} N, i_{*}^{y} \mathbb{Z}\right)$ (using $i^{y}: y \rightarrow X$ ).

The former has already been done (Theorem 3.16). For the latter we obtain a spectral sequence

$$
\operatorname{Ext}_{x}^{p}\left[N, R^{q}\left(i^{\prime}\right)^{\prime}\left(i_{*}^{\prime} \mathbb{Z}\right)\right] \Rightarrow \operatorname{Ext}_{X}^{p+q}\left(i_{*}^{\prime} N, i_{*}^{\prime} \mathbb{Z}\right)
$$

Now $\left[R^{q}\left(i^{\prime}\right)^{\prime}\right]\left(i_{*}^{\prime} \mathbb{Z}\right)=R^{q}\left[\left(i^{\prime}\right)^{\prime} i_{*}^{y}\right] \mathbb{Z}$. But $\left(i^{\prime}\right)^{\prime} i_{*}^{*}=0$, so we have reduced the original Yoneda pairing to the equivalent

$$
\operatorname{Ext}_{\tilde{X}}^{p}\left(i_{*} \mathbb{Z}, i_{*} M\right) \times \operatorname{Ext}_{\bar{X}}^{2-p}\left(i_{*} M, i_{*} \mathbb{Z}\right) \rightarrow \operatorname{Ext}_{\tilde{X}}^{2}\left(i_{*} \mathbb{Z}, i_{*} \mathbb{Z}\right) .
$$

Finally, by exactness of $i_{*}$, the fact that $i^{*} i_{*}$ is the identity, and the naturality of the Yoneda pairing, this is isomorphic to the pairing

$$
\operatorname{Ext}_{x}^{p}(\mathbb{Z}, M) \times \operatorname{Ext}_{x}^{2-p}(M, \mathbb{Z}) \rightarrow \operatorname{Ext}_{x}^{2}(\mathbb{Z}, \mathbb{Z})
$$

in the $G^{x}$-module category.

Theorem 4.2 (Punctual Duality Theorem). Let $P$ be a punctual finite sheaf on $X$ concentrated on finite primes. Then the Yoneda pairing

$$
H^{p}(\tilde{X}, P) \times \operatorname{Ext}_{\tilde{X}}^{3-p}\left(P, \phi_{*} G_{m}\right) \rightarrow H^{3}\left(X, \phi_{*} G_{m}\right)
$$

is a perfect duality of finite groups. $H^{p}(\tilde{X}, P)$ vanishes for $p>1$ and $\operatorname{Ext}_{\tilde{X}}^{q}\left(P, \phi_{*} G_{m}\right)$ vanishes for $q>3$.

Proof. By the preceding proposition this is equivalent to the corresponding statement about the pairing

$$
H^{p}(\hat{\mathbb{Z}}, M) \times \operatorname{Ext}_{\hat{\mathbb{Z}}}^{2-p}(M, \mathbb{Z}) \rightarrow H^{2}(\hat{\mathbb{Z}}, \mathbb{Z}) .
$$

But this is the same as

$$
H^{p}(\hat{\mathbb{Z}}, M) \times \operatorname{Ext}_{\hat{\mathbf{Z}}}^{1-p}(M, \mathbb{Q} / \mathbb{Z}) \rightarrow H^{1}(\hat{\mathbb{Z}}, \mathbb{Q} / \mathbb{Z})
$$

and to say that this is a perfect duality is the same as saying that $\mathbb{Q} / \mathbb{Z}$ is a dualizing module for $\hat{\mathbb{Z}}$ (see [7, pp. I-27 and I-31]). 
5. Proof of the duality theorem. As in other versions of this theorem, we begin with a constructible sheaf on $X$. Recall that a sheaf is constructible if there is an open subscheme $U$ of $X$ such that the pullbacks of $F$ to $U$ and to each point in its closed complement are locally constant sheaves whose values are finite Abelian groups.

TheOREM 5.1 (ARTIN-Verdier DUALITY ON $\tilde{X}$ ). If $F$ is constructible, then

(a) $H^{p}\left(\tilde{X}, \phi_{*} F\right)=\operatorname{Ext}_{\tilde{X}}^{p}\left(\phi_{*} F, \phi_{*} G_{m}\right)=0$ for $p>3$.

(b) The Yoneda pairing

$$
H^{p}\left(\tilde{X}, \phi_{*} F\right) \times \operatorname{Ext}_{\tilde{X}}^{3-p}\left(\phi_{*} F, \phi_{*} G_{m}\right) \rightarrow H^{3}\left(\tilde{X}, \phi_{*} G_{m}\right)
$$

is a perfect duality of finite groups for $p \geqslant 2$.

(c) If, for each $x_{i} \in X_{\infty}$, the group $I_{i}$ acts trivially on the $G_{K}$-module $j^{*} F,(5.2)$ is perfect for all $p$.

We will prove this from Zink's theorem, so we need to define the modified cohomology which is used in that result. Once again, our statements include the complex primes of $\tilde{X}$, but this in no essential way affects the results.

Definitions 5.3. (a) If $F$ is a sheaf on an open subscheme $U$ of $X$, then $\hat{F}$ on $\tilde{U}$ is the sheaf $\left(\left\{H_{0}\left(I_{i}, j^{*} F\right)\right\}, F,\left\{N_{i}\right\}\right)$, where $N_{i}: H_{0}\left(I_{i}, j^{*} F\right) \rightarrow H^{0}\left(I_{i}, j^{*} F\right)$ is the usual norm map linking homology with cohomology. Note that $\hat{F}$ agrees with $\phi^{*} F$ at finite primes and at complex primes.

(b)

$$
\hat{H}^{p}(U, F)= \begin{cases}H^{p}(\tilde{U}, \hat{F}), & p \geqslant 0, \\ \bigoplus_{x_{i} \in X_{\infty}} H^{p-1}\left(I_{i}, j^{*} F\right), & p<0 .\end{cases}
$$

For $p<0, \hat{H}^{p}$ is the usual negative-dimensional group cohomology.

THEOREM $5.4[9,3.2]$. The Yoneda pairing

$$
\hat{H}^{p}(X, F) \times \operatorname{Ext}_{X}^{3-p}\left(F, G_{m}\right) \rightarrow \hat{H}^{3}\left(X, G_{m}\right) \cong \mathbb{Q} / \mathbb{Z}
$$

is a perfect duality of finite groups.

To prove 5.1, first note that for any sheaf $F$ on $X$ there is natural map $\psi$ : $\hat{F} \rightarrow \phi_{*} F$ of sheaves on $\tilde{X}$ induced by the norm at infinite primes. In nonnegative dimensions this induces a map $\psi: \hat{H}^{p}(X, F) \rightarrow H^{p}\left(\tilde{X}, \phi_{*} F\right)$. For $p \geqslant 2$, this is an isomorphism; this follows from the facts that $\hat{F}$ and $\phi_{*} F$ agree on $X$ and that the cohomology of sheaves concentrated on $X_{\infty}$ vanishes in positive dimensions.

One consequence is that $\hat{H}^{3}\left(X, G_{m}\right) \cong H^{3}\left(\tilde{X}, \phi_{*} G_{m}\right)$; another is that 5.1(a) is proven for $H^{p}$. To complete part (a), first assume that $F$ is punctual. Then $\phi_{*} F$ is punctual, concentrated on $X$, so $\operatorname{Ext}_{\tilde{X}}^{p}\left(\phi_{*} F, \phi_{*} G_{m}\right)$ vanishes for $p>3$ by our punctual duality theorem (4.2). We may thus assume by the usual decomposition of constructible sheaves that $F=\rho_{!} D$ for some locally constant sheaf $D$ on a subscheme $U$ of $X$. If $\rho$ denotes both inclusions $U \rightarrow X$ and $\tilde{U} \rightarrow \tilde{X}$, we may write

$$
\operatorname{Ext}_{\tilde{X}}^{q}\left(\phi_{*} \rho ! D, \phi_{*} G_{m}\right)=\operatorname{Ext}_{\tilde{X}}^{q}\left(\rho ! \phi_{*} D, \phi_{*} G_{m}\right) \cong \operatorname{Ext}_{\tilde{U}}^{q}\left(\phi_{*} D, \phi_{*} G_{m}\right) \text {. }
$$

We use the spectral sequence

$$
H^{p}\left(\tilde{U}, \underline{\operatorname{Ext}}_{\tilde{U}}^{q}\left(\phi_{*} D, \phi_{*} G_{m}\right)\right) \Rightarrow \operatorname{Ext}_{\tilde{U}}^{p+q}\left(\phi_{*} D, \phi_{*} G_{m}\right)
$$


and, as in [6, Proposition C, or 9, 3.2.2], we discover that the local Ext ${ }^{q}$ sheaves are 0 for $q>1$ and punctual for $q=1$. For $p+q>3$ and $q>0$, such sheaves have no $p$-cohomology, leaving $H^{p}\left(\tilde{U}, \operatorname{Hom}_{\tilde{U}}\left(\phi_{*} D, \phi_{*} G_{m}\right)\right)$ as the only terms on the left of (5.6). These Hom sheaves are constructible in the sense of Zink: constructible on $U$ and finite at $\overline{X_{\infty}}$; so by [9, Theorem 2.6] their cohomology is zero in dimensions $\geqslant 4$. The entire sequence (5.6) thus vanishes.

Now let $\psi^{\prime}: \operatorname{Ext}_{\tilde{X}}^{q}\left(\phi_{*} F, \phi_{*} G_{m}\right) \rightarrow \operatorname{Ext}_{X}^{q}\left(F, G_{m}\right)$ be the map induced by the inclusion $\phi_{!} F \rightarrow \phi_{*} F$, recalling the identification $\operatorname{Ext}_{\tilde{X}}^{q}\left(\phi_{!} F, \phi_{*} G_{m}\right) \cong \operatorname{Ext}_{X}^{q}\left(F, G_{m}\right)$ coming from the exact adjoint pair $\left(\phi_{!}, \phi^{*}\right)$. We claim that the diagram

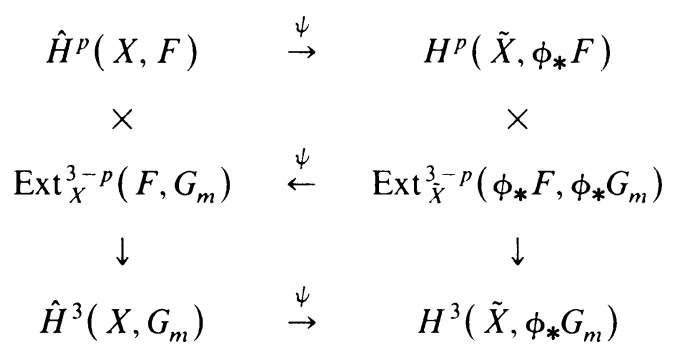

is commutative for $p>0$, in the sense $\psi a \times b=\psi\left(a \times \psi^{\prime} b\right)$, for $a \in \hat{H}^{p}(X, F)$ and $b \in \operatorname{Ext}_{\tilde{X}}^{q}\left(\phi_{*} F, \phi_{*} G_{m}\right)$. For $p=0$ we shall show that the diagram commutes under the additional hypothesis that each $I_{i}$ acts trivially on $j^{*} F$.

To prove this claim, note that (5.7) is trivial for $p>3$. If $p=3$, an element $b \in \operatorname{Ext}_{\tilde{X}}^{3-p}\left(\phi_{*} F, \phi_{*} G_{m}\right)=\operatorname{Hom}_{\tilde{X}}\left(\phi_{*} F, \phi_{*} G_{m}\right)$ is induced from a map $\beta$ : $\operatorname{Hom}_{X}\left(F, G_{m}\right)$ by the adjunction of $\phi^{*}$ and $\phi_{*}$. Clearly $\psi^{\prime} b=\beta$; also the map $b$ : $H^{3}\left(\tilde{X}, \phi_{*} F\right) \rightarrow H^{3}\left(\tilde{X}, \phi_{*} G_{m}\right)$ on cohomology is the same as that induced by $\beta$, considering $H^{3}\left(\tilde{X}, \phi_{*} \cdot\right)$ as a composite functor. Examining the Yoneda pairings for Ext-dimension 0 , the commutativity of (5.7) for our element $b$ reduces to that of

$$
\begin{array}{ccc}
\hat{H}^{3}(X, F) & \stackrel{\psi}{\rightarrow} & H^{3}\left(\tilde{X}, \phi_{*} F\right) \\
\downarrow \beta & & \downarrow \beta \\
\hat{H}^{3}\left(X, G_{m}\right) & \stackrel{\psi}{\rightarrow} & H^{3}\left(\tilde{X}, \phi_{*} G_{m}\right) .
\end{array}
$$

This simply says that $\psi$ is a natural transformation of functors on $\mathscr{S}_{X}$, which is clear.

For $p=1,2$ we shift dimensions in the bottom two lines of (5.7) by beginning the construction of injective resolutions of $G_{m}$ and $\phi_{*} G_{m}$, but we must take care that these are compatible with the pairings in the two different categories $\mathscr{S}_{X}$ and $\mathscr{S}_{\tilde{X}}$.

Let $G_{m} \rightarrow J_{1}$ be an embedding into an injective of $\mathscr{S}_{X}$. Then $\phi_{*} G_{m} \rightarrow \phi_{*} J_{1}$ is an embedding into an injective, and if $I_{1}=\phi_{*} J_{1}, I_{1}$ has the property $I_{1} \cong \phi_{*} \phi^{*} I_{1}$ because $\phi^{*} \phi_{*}$ is the identity functor. Let $D_{1}=\operatorname{coker}\left(\phi_{*} G_{m} \rightarrow I_{1}\right)$. We have the exact sequences

$$
0 \rightarrow \phi_{*} G_{m} \rightarrow I_{1} \rightarrow D_{1} \rightarrow 0
$$

and

$$
0 \rightarrow G_{m} \rightarrow \phi^{*} I_{1} \rightarrow \phi^{*} D_{1} \rightarrow 0
$$


since $\phi^{*}$ is exact. If we apply $\phi_{*}$ to (5.10) we get a sequence

$$
0 \rightarrow \phi_{*} G_{m} \rightarrow \phi_{*} \phi^{*} I_{1} \rightarrow \phi_{*} \phi^{*} D_{1} \rightarrow 0
$$

which is exact because $R^{1} \phi_{*} G_{m}=0$. (This is true since every element of $H^{1}\left(X, G_{m}\right)=$ Pic $A$ vanishes in an extension which is unramified even at infinite primes.)

Comparing (5.9) and (5.11) we discover that the natural map $D_{1} \rightarrow \phi_{*} \phi^{*} D_{1}$ is an isomorphism.

We continue by embedding $\phi^{*} D_{1}$ into a injective $J_{2}$ and putting $I_{2}=\phi_{*} J_{2}$, $D_{2}=\operatorname{coker}\left(\phi_{*} \phi^{*} D_{1} \rightarrow I_{2}\right)$. Taking into account $D_{1} \cong \phi_{*} \phi^{*} D_{1}$, we have exact sequences

$$
0 \rightarrow D_{1} \rightarrow I_{1} \rightarrow D_{2} \rightarrow 0
$$

and

$$
0 \rightarrow \phi^{*} D_{1} \rightarrow \phi^{*} I_{1} \rightarrow \phi^{*} D_{2} \rightarrow 0 .
$$

Applying $\phi_{*}$ to (5.13) and using the Five-Lemma, we discover that $D_{2} \rightarrow \phi_{*} \phi^{*} D_{2}$ is a monomorphism.

Since $\hat{H}$ is a cohomology functor on $\mathscr{S}_{X}$, we may use (5.10) and (5.13) to show $\hat{H}^{3}\left(X, G_{m}\right) \cong \hat{H}^{2}\left(X, \phi^{*} D_{1}\right) \cong H^{1}\left(X, \phi^{*} D_{2}\right)$ (see $\left.[9,3.1 .4 .1]\right)$. Similarly $H^{3}\left(\tilde{X}, \phi_{*} G_{m}\right)$ $\cong H^{2}\left(\tilde{X}, D_{1}\right) \cong H^{1}\left(\tilde{X}, D_{2}\right)$. To relate these isomorphisms to the map $\psi$, note that we have $\psi: \widehat{\phi^{*} I_{1}} \rightarrow \phi_{*} \phi^{*} I_{i}=I_{i}$ for $i=1,2$ and $\psi: \overline{\phi^{*} D_{1}} \rightarrow \phi_{*} \phi^{*} D_{1}=D_{1}$. We also have $\psi: \overline{\phi^{*} D_{2}} \rightarrow D_{2}$ by the properties of cokernels (recall $(\hat{\imath})$ is right exact). Each of our maps $\psi$ is the identity when restricted to $\mathscr{S}_{X}$.

Under our identifications the map $\psi$ on $\hat{H}^{3}\left(X, G_{m}\right)$ becomes $\psi: \hat{H}^{p}\left(X, \phi^{*} D_{3-p}\right)$ $\rightarrow H^{p}\left(\tilde{X}, D_{3-p}\right)$.

We also have

$$
\operatorname{Ext}_{X}^{q}\left(F, G_{m}\right) \cong \operatorname{coker}\left(\operatorname{Hom}_{X}\left(F, \phi^{*} I_{q}\right) \rightarrow \operatorname{Hom}_{X}\left(F, \phi^{*} D_{q}\right)\right)
$$

and

$$
\operatorname{Ext}_{\tilde{X}}^{q}\left(\phi_{*} F, \phi_{*} G_{m}\right) \cong \operatorname{coker}\left(\operatorname{Hom}_{\tilde{X}}\left(\phi_{*} F, I_{q}\right) \rightarrow \operatorname{Hom}_{\tilde{X}}\left(\phi_{*} F, D_{q}\right)\right)
$$

for $q=1,2$. These identifications commute with the functor $\psi^{\prime}$. By the dimensionshifting properties of the Yoneda pairing, (5.7) will be commutative once we show the same is true for

$$
\begin{array}{ccc}
\hat{H}^{p}(X, F) & \stackrel{\psi}{\rightarrow} & H^{p}\left(\tilde{X}, \phi_{*} F\right) \\
\times & & \times \\
\operatorname{Hom}_{X}\left(F, \phi^{*} D_{q}\right) & \stackrel{\psi^{\prime}}{\leftarrow} & \operatorname{Hom}_{\tilde{X}}\left(\phi_{*} F, D_{q}\right) \\
\downarrow & & \downarrow \\
\hat{H}^{p}\left(X, \phi^{*} D_{q}\right) & \stackrel{\psi}{\rightarrow} & H^{p}\left(\tilde{X}, D_{q}\right)
\end{array}
$$


Choose $b \in \operatorname{Hom}_{\tilde{X}}\left(\phi_{*} F, D_{q}\right)$, and let $\beta=\psi^{\prime} b$. If we rewrite $\hat{H}^{p}(X, F)=$ $H^{p}(\tilde{X}, \hat{F})$, etc., it suffices to show that

$$
\begin{array}{ccc}
H^{p}(\tilde{X}, \hat{F}) & \stackrel{\psi}{\rightarrow} & H^{p}\left(\tilde{X}, \phi_{*} F\right) \\
\hat{\beta} \downarrow & & \downarrow b \\
H^{p}\left(\tilde{X}, \widehat{\phi^{*} D_{q}}\right) & \stackrel{\psi}{\rightarrow} & H^{p}\left(\tilde{X}, D_{q}\right)
\end{array}
$$

commutes, where $\hat{\beta}$ has the obvious meaning. Each map in 5.14 is now induced by a map of sheaves in $\mathscr{S}_{\tilde{X}}$. Both maps $\psi$ are the identity on $\mathscr{S}_{X}$, and $b$ and $\hat{\beta}$ agree on $\mathscr{S}_{X}$. Both ways around the square (in $\mathscr{S}_{\tilde{X}}$ ) give maps $\hat{F} \rightrightarrows D_{q}$ which agree on $\mathscr{S}_{X}$. The map $D_{q} \rightarrow \phi_{*} \phi^{*} D_{q}$ is also the identity on $\mathscr{S}_{X}$; since we have shown that this is a monomorphism it suffices to show that the composites $\hat{F} \rightrightarrows D_{q} \rightarrow \phi_{*} \phi^{*} D_{q}$ are the same. But $\operatorname{Hom}_{\tilde{X}}\left(\hat{F}, \phi_{*} \phi^{*} D_{q}\right)=\operatorname{Hom}_{X}\left(\phi^{*} \hat{F}, \phi^{*} D_{q}\right)=\operatorname{Hom}_{X}\left(F, \phi^{*} D_{q}\right)$; i.e., there is only one way of extending a map $F \rightarrow \phi^{*} D_{q}$ to $\hat{F} \rightarrow \phi_{*} \phi^{*} D_{q}$, and we have our commutativity for $p>0$.

We shift dimensions differently for $p=0$, using the sequence

$$
0 \rightarrow \phi_{*} G_{m} \rightarrow \alpha_{*} G_{m} \rightarrow \amalg i_{*}^{x} \mathbb{Z} \rightarrow 0,
$$

which is (2.3). We show that the assumption of trivial action by the $I_{i}$ implies $\operatorname{Ext}_{\tilde{X}}^{3}\left(\phi_{*} F, \alpha_{*} G_{m}\right)=0$.

Since $F$ is constructible there is an open inclusion $\rho: U \rightarrow X$ and an exact sequence $0 \rightarrow \phi_{*} \rho_{!} \rho^{*} F \rightarrow \phi_{*} F \rightarrow P \rightarrow 0$ where $P$ is punctual and concentrated at finite primes, and $\rho^{*} F$ is locally constant on $U$. Note that for such $P, P=\phi_{!} \phi^{*} P$ so that $\operatorname{Ext}_{\tilde{X}}^{p}\left(P, \alpha_{*} G_{m}\right)=\operatorname{Ext}_{X}^{p}\left(\phi^{*} P, j_{*} G_{m}\right)$ by the usual adjunction and exactness. This last Ext group is zero, as we have seen in the proof of 3.16 , so our statement regarding $\mathrm{Ext}^{3}$ reduces to the same statement for $\phi_{*} \rho_{!} \rho^{*} F$. A locally constant sheaf is induced from the $G_{K}$-module $j^{*} F$ (see [6]), so by replacing $P$ with another punctual sheaf we reduce to $\operatorname{Ext}_{\tilde{X}}^{3}\left(\phi_{*} j_{*} j^{*} F, \alpha_{*} G_{m}\right)$.

Let $L / K$ be an extension such that $G_{L}$ acts trivially on $j^{*} F$, let $Y=\operatorname{Spec}($ integers of $L$ ), and let $\pi: \tilde{Y} \rightarrow \tilde{X}$ be the natural projection. The special assumption on $F$ implies that each $I_{i} \subseteq G_{L}$ and therefore that $Y_{\infty}$ is unramified over $\tilde{X}$. There is a monomorphism $j_{*} j^{*} F \rightarrow \pi_{*} \pi^{*} j_{*} j^{*} F$ in $\mathscr{S}_{X}$, coming from the standard map $j^{*} F \rightarrow$ $M_{G_{l}}^{G_{K}} j^{*} F[7$, p. I-12].

The cokernel $C$ of this map is constructible [6]. Since $\pi_{*} \pi^{*} j_{*} j^{*} F$ is constant on $Y$, and $\tilde{Y} \rightarrow \tilde{X}$ is unramified over $X_{\infty}, \phi_{*} \pi_{*} \pi^{*} J_{*} J^{*} F \rightarrow \phi_{*} C$ is surjective on $\tilde{X}$. Putting $G=j_{*} j^{*} F$, we thus have an exact sequence $0 \rightarrow \phi_{*} G \rightarrow \pi_{*} \pi^{*} \phi_{*} G \rightarrow \phi_{*} C \rightarrow 0$, and we will get our statement about $\phi_{*} G$ by showing that both $\operatorname{Ext}_{\tilde{X}}^{3}\left(\pi_{*} \pi^{*} \phi_{*} G, \alpha_{*} G_{m}\right)$ and $\operatorname{Ext}_{\tilde{X}}^{4}\left(\phi_{*} C, \alpha_{*} G_{m}\right)$ are zero. By the norm theorem for $\alpha_{*} G_{m}$ (3.16), the former of these is isomorphic to $\operatorname{Ext}_{\tilde{Y}}^{3}\left(\pi^{*} \phi_{*} G, \alpha_{*} G_{m}\right)$, and since $\pi^{*} \phi_{*} G$ is constant we reduce to $\operatorname{Ext}_{\bar{Y}}^{3}\left(\mathbb{Z} / n \mathbb{Z}, \alpha_{n} G_{m}\right)$.

Using $0 \rightarrow \mathbb{Z} \stackrel{n}{\rightarrow} \mathbb{Z} \rightarrow \mathbb{Z} / n \mathbb{Z} \rightarrow 0$ and Proposition 2.5 , this is zero by the divisibility of local Brauer groups. As for $\phi_{*} C$, our proof that $\operatorname{Ext}_{\tilde{X}}^{4}\left(\phi_{*} C, \phi_{*} G_{m}\right)=0$ works just as well for $\alpha_{*} G_{m}$. 
We may now perform our dimension-shifting. Let $E_{1}$ be the sheaf $\amalg i_{*}^{x} \mathbb{Z}$ on $X$. Since $j^{*} E_{1}=0$, we have that on $\tilde{X}, \hat{E}_{1}=\phi_{*} E_{1}=\amalg i_{*}^{x} \mathbb{Z}$, where $i^{x}$ denotes the inclusion into $\tilde{X}$ as well.

There is thus a map of exact sequences

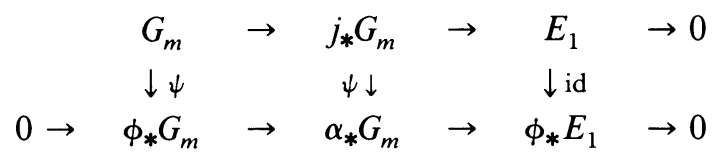

and since both $\operatorname{Ext}_{\tilde{X}}^{3}\left(\phi_{*} F, \alpha_{*} G_{m}\right)$ and $H^{3}\left(\tilde{X}, \phi_{*} G_{m}\right)$ vanish, it suffices (similarly to the case $p>0$ ) to prove the commutativity of

$$
\begin{array}{ccc}
\hat{H}^{0}(X, F) & \stackrel{\psi}{\rightarrow} & H^{0}\left(\tilde{X}, \phi_{*} F\right) \\
\times & & \times
\end{array}
$$

$$
\begin{array}{ccc}
\operatorname{Ext}_{X}^{2}\left(F, E_{1}\right) & \stackrel{\psi^{\prime}}{\leftarrow} & \operatorname{Ext}_{X}^{2}\left(\phi_{*} F, \phi_{*} E_{1}\right) \\
\downarrow & & \downarrow \\
\hat{H}^{2}\left(X, E_{1}\right) & \stackrel{\psi}{\psi=\text { id }} & H^{2}\left(\tilde{X}, \phi_{*} E_{1}\right) .
\end{array}
$$

Because $E_{1}$ has the property $j^{*} E_{1}=0$, and because each $i_{*}^{x}$ preserves injectives, we may embed $E_{1}$ in an injective $Q_{1}$ which has the property $\hat{Q}_{1}=\phi_{*} Q_{1}=\phi ! Q_{1}$.

The cokernel $E_{2}$ of this embedding has the same property. Repeating this process, we have maps of exact sequences

$$
\begin{array}{rrrr}
0 \rightarrow \hat{E}_{i} & \rightarrow \hat{Q}_{i} & \rightarrow \hat{E}_{i+1} & \rightarrow 0 \\
=\downarrow & =\downarrow & =\downarrow & \\
0 \rightarrow \phi_{*} E_{i} & \rightarrow \phi_{*} Q_{i} & \rightarrow \phi_{*} E_{i+1} & \rightarrow 0
\end{array}
$$

for $i=1,2$, and we use these to reduce (5.15) to

$$
\begin{array}{ccc}
\hat{H}^{0}(X, F) & \stackrel{\psi}{\rightarrow} & H^{0}\left(\tilde{X}, \phi_{*} F\right) \\
\times & & \times \\
\operatorname{Hom}_{X}\left(F, E_{3}\right) & \stackrel{\psi^{\prime}}{\leftarrow} & \operatorname{Hom}_{\tilde{X}}\left(\phi_{*} F, \phi_{*} E_{3}\right) \\
\downarrow & & \downarrow \\
\hat{H}^{0}\left(X, E_{3}\right) & \stackrel{\psi}{\rightarrow} & H^{0}\left(\tilde{X}, \phi_{*} E_{3}\right) .
\end{array}
$$

As before, an element $b \in \operatorname{Hom}_{\tilde{X}}\left(\phi_{*} F, \phi_{*} E_{3}\right)$ with $\psi^{\prime} b=\beta$ gives

$$
\begin{array}{ccc}
\hat{F} & \rightarrow & \phi_{*} F \\
\hat{\beta} \downarrow & & \downarrow b \\
\hat{E}_{3} & =\phi_{*} E_{3}
\end{array}
$$

and in this case we may conclude simply with the identity $\operatorname{Hom}_{\tilde{X}}\left(F, \phi_{*} E_{3}\right) \cong$ $\operatorname{Hom}_{X}\left(F, E_{3}\right)$. 
We may now use the commutativity of (5.7) to draw conclusions about the pairing for $\phi_{*} F$ from information about the pairing for the modified cohomology. For $p \geqslant 2$, both $\psi$ and $\psi^{\prime}$ are isomorphisms; we have mentioned this for $\psi$, and for $\psi^{\prime}$ it follows from the fact that the Ext-functors vanish on the cokernel of $\phi_{!} F \rightarrow \phi_{*} F$ in dimensions $<3$ (Lemma 3.8). Part (b) of Theorem 5.1 follows immediately. For part (c), let $M$ be the Abelian group underlying the $G_{K}$-module $j^{*} F$, and assume each $I_{i}$ acts trivially on $M$. The map $\psi: \hat{F} \rightarrow \phi_{*} F$ may be decomposed into the exact sequences

$$
0 \rightarrow P_{1} \rightarrow \hat{F} \rightarrow I \rightarrow 0, \quad P_{1}=\bigoplus_{x_{i} \text { real }} \kappa_{i *}\left({ }_{2} M\right)
$$

and

$$
0 \rightarrow I \rightarrow \phi_{*} F \rightarrow P_{2} \rightarrow 0, \quad P_{2}=\bigoplus \kappa_{i *}(M / 2 M),
$$

because of the trivial action. Also for this reason we have

$$
0 \rightarrow \phi_{!} F \rightarrow \phi_{*} F \rightarrow P_{3} \rightarrow 0, \quad P_{3}=\kappa_{*} \kappa^{*} \phi_{*} F=\bigoplus_{x_{i} \in X_{\infty}} M
$$

We will show that there is a commutative diagram of exact sequences

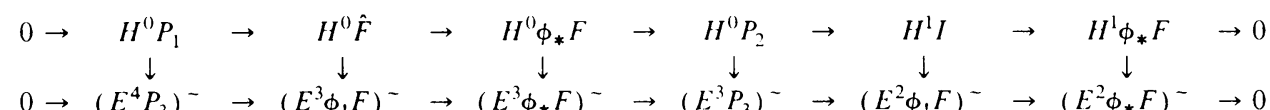

in which $H^{p}$ stands for $H^{p}(\tilde{X}, \cdot), E^{p}$ stands for $\operatorname{Ext}_{\tilde{X}}^{p}\left(\cdot, \phi_{*} G_{m}\right)$ and $(\cdot)^{\sim}$ denotes Pontrjagin dual.

The bottom sequence is the dual of the long exact $E^{p}$-sequence of (5.18), by 3.8 and by part (a) of Theorem 5.1. (The finiteness of $E^{p} \phi_{*} F$ for all $p$ and any constructible $F$ follows.) The top sequence comes from taking cohomology in (5.16) and (5.17) and recalling that $H^{p} P_{1}=H^{p} P_{2}=0$ for $p>0$. The vertical inaps on $H^{0} \hat{F}, H^{0} \phi_{*} F$, and $H^{1} \phi_{*} F$ come from the Yoneda pairing, so that the second square is commutative.

We may define the vertical map on $H^{0} P_{1}$ by restriction from $H^{0} F$, so that the first square commutes. Since the map on $H^{0} \hat{F}$ is an isomorphism, this map is injective; but 3.8 shows that $H^{0} P_{1}$ and $E^{4} P_{3}$ have the same order, so our first two vertical arrows are isomorphisms.

On $H^{0} P_{2}$, note that the map $P_{3} \rightarrow P_{2}$ defined as zero at complex primes and projection modulo 2 at real primes induces an isomorphism $\left(E^{3} P_{3}\right)^{\sim} \rightarrow\left(E^{3} P_{2}\right)^{\sim}$ by 3.8. Define the vertical map via the Yoneda pairing $H^{0} P_{2} \times E^{3} P_{2} \rightarrow H^{3} \phi_{*} G_{m}$. To show that the third square commutes, we may write $\phi^{*} F \rightarrow P_{2}$ as a composite $\phi_{*} F \rightarrow P_{3} \rightarrow P_{2}$, where the first arrow comes from (5.18). There is thus a commutative diagram

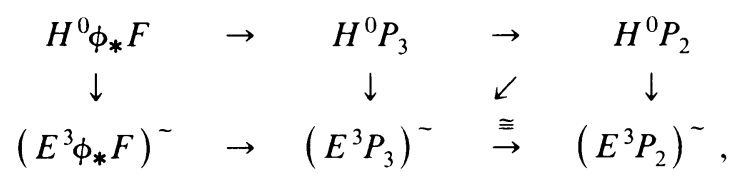


in which the squares commute by the naturality of the Yoneda pairing, and the diagonal arrow is defined via the isomorphism in the lower right.

We also claim $H^{0} P_{2} \rightarrow\left(E^{3} P_{3}\right)^{\sim}$ s an isomorphism. The cohomology of $P_{2}$ is equal to its "compact" cohomology at real primes (see 1.4), and there is a diagram

$$
\begin{aligned}
& \oplus \operatorname{Hom}(\mathbb{Z}, M / 2 M) \cong \oplus H_{x_{i}}^{0} P_{2} \stackrel{\cong}{\rightarrow} \quad H^{0} P_{2} \\
& \begin{array}{ccccc}
\times & & \times & & \times \\
\oplus \operatorname{Hom}(M / 2 M, \mathbb{Z} / 2 \mathbb{Z}) & \cong & E^{3} P_{2} & = & E^{2} P_{2} \\
\downarrow & & \downarrow & & \downarrow \\
\oplus \operatorname{Hom}(\mathbb{Z}, \mathbb{Z} / 2 \mathbb{Z}) & \cong & \oplus H_{x_{i}}^{3} \phi_{*} G_{m} & \rightarrow & H^{3} \phi_{*} G_{m} .
\end{array}
\end{aligned}
$$

The right half is a commutative diagram of pairings because $H_{x_{i}}^{p}(\tilde{X}, \cdot)=$ $\operatorname{Ext}_{\tilde{X}}^{p}\left(\kappa_{i *} \mathbb{Z}, \cdot\right)$ and the top and bottom maps come from $\mathbb{Z} \rightarrow \kappa_{i *} \mathbb{Z}$. The left half commutes because each element of $\operatorname{Hom}_{\tilde{X}}\left(\kappa_{i *} \mathbb{Z}, P_{2}\right)=\operatorname{Hom}(\mathbb{Z}, M / 2 M)$ induces a "map of spectral sequences" from

$$
\operatorname{Ext}^{p}\left(M / 2 M, R^{q} \kappa_{i}^{\prime} \phi_{*} G_{m}\right) \Rightarrow \operatorname{Ext}_{\tilde{X}}^{p+q}\left(\kappa_{i *} M / 2 M, \phi_{*} G_{m}\right)
$$

to

$$
\operatorname{Ext}^{p}\left(\mathbb{Z}, R^{q} \kappa_{i}^{\vdots} \phi_{*} G_{m}\right) \Rightarrow \operatorname{Ext}_{\tilde{X}}^{p+q}\left(\kappa_{i *} \mathbb{Z}, \phi_{*} G_{m}\right) .
$$

The diagram thus shows that the pairing is simply that of Homs on 2-torsion Abelian groups for each real prime.

Next, we define $H^{1} I \rightarrow\left(E^{2} \phi_{!} F\right)$ via the isomorphism $H^{1} \hat{F} \rightarrow H^{1} I$ coming from (5.16). This pairing map is an isomorphism by 5.4, and the last square in (5.19) clearly commutes. All of the vertical maps are isomorphisms with the possible exceptions of those on the cohomology of $\phi_{*} F$; we will have proven these to be isomorphisms as well as once we show that the fourth square commutes.

For this purpose we may precede the maps in that square with the surjective map $H^{0} P_{3} \rightarrow H^{0} P_{2}$. We want to show $H^{0} P_{3} \rightarrow H^{0} P_{2} \rightarrow H^{1} I \rightarrow\left(E^{2} \phi_{!} F\right)^{-}$is the same as $H^{0} P_{3} \rightarrow H^{0} P_{2} \rightarrow\left(E^{3} P_{3}\right)^{\sim} \rightarrow\left(E^{2} \phi_{!} F\right)^{\sim}$. By mapping (5.18) to (5.17) we get a commutative square

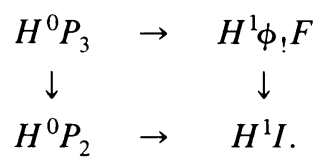

If we replace $H^{1} I$ in this square by the isomorphic $H^{1} \hat{F}$, the right-hand vertical map is replaced by the map on cohomology coming from the inclusion $\phi_{!} F \rightarrow \hat{F}$, because the composite $\phi_{!} F \rightarrow \hat{F} \rightarrow I$ is the same as the map connecting (5.18) and (5.17). We may thus replace

$$
H^{0} P_{3} \rightarrow H^{0} P_{2} \rightarrow H^{1} I \stackrel{\cong}{\rightarrow} H^{1} \hat{F} \rightarrow\left(E^{2} \phi_{!} F\right)^{\sim}
$$

by

$$
H^{0} P_{3} \rightarrow H^{1} \phi_{!} F \rightarrow H^{1} \hat{F} \rightarrow\left(E^{2} \phi, F\right)^{\sim} .
$$


But the composite of the last two arrows is the Yoneda pairing on $\phi_{!} F$, because $H^{p} \phi, F \rightarrow \hat{H}^{p} F$ is a map of cohomology functors on $F$. Thus by shifting dimensions in the pairing, using (5.18), we may replace the last sequence by $H^{0} P_{3} \rightarrow\left(E^{3} P_{3}\right)^{\sim} \rightarrow$ $\left(E^{2} \phi, F\right)^{-}$. Our map on $H^{0} P_{2}$ fits into the first of these arrows, and the proof is complete.

REMARK (5.22). Our duality theorem restores the pairing

$$
H^{1} \mathbb{Z} / n \mathbb{Z} \times \operatorname{Pic} A / n \operatorname{Pic} A \rightarrow \mathbb{Q} / \mathbb{Z}
$$

for even $n$ (see 2.13) which is missing from Zink's more general version. This is one reason why it may be useful to have both theorems.

AcKnowledgement. The author wishes to thank Professor Stephen Lichtenbaum for his consistent encouragement, for his careful reading of the manuscript, and for his suggestions which led to a more complete and useful article.

\section{REFERENCES}

1. M. Artin, Grothendieck topologies, mimeographed notes, Harvard Univ., 1962.

2. M. Artin and J. L. Verdier, Seminar on étale cohomology of number fields, Lecture Notes of Summer Institute on Algebraic Geometry, Woods Hole, 1964.

3. M. Bienenfeld, Values of zeta- and L-functions at zero: The case of a non-totally imaginary algebraic number field, Ph.D. dissertation, Cornell Univ., 1982.

4. S. Lichtenbaum, Values of zeta- and L-functions at zero, Soc. Math. France Astérisque 24-25 (1975).

5. S. Lichtenbaum and M. Bienenfeld, Values of zeta- and L-functions at zero: a cohomological characterization (to appear).

6. B. Mazur, Etale cohomology of number fields, Ann. Sci. École Norm. Sup. 6 (1973).

7. J.-P. Serre, Cohomologie galoisienne, Lecture Notes in Math., vol. 5, Springer-Verlag, 1964.

8. J. Tate, The cohomologv groups of algebraic number fields, Proc. Internat. Congress Math., Amsterdam, 1954.

9. T. Zink, Étale cohomologv and duality in number fields, Haberland, Galois cohomologv, Berlin, 1978. Appendix 2.

Department of Mathematics and Computer Science, St. John's University, Jamaica, New YORK 11439 